University of Wollongong

Research Online

Faculty of Social Sciences - Papers (Archive) Faculty of Arts, Social Sciences \& Humanities

2014

Sequential processing in the equiprobable auditory Go/NoGo task: children vs. adults

Robert Barry

University of Wollongong, rbarry@uow.edu.au

Frances M. De Blasio

University of Wollongong, francesd@uow.edu.au

Jay P. Borchard

Univeristy Of Wollongong, jb893@uowmail.edu.au

Follow this and additional works at: https://ro.uow.edu.au/sspapers

Part of the Education Commons, and the Social and Behavioral Sciences Commons

Research Online is the open access institutional repository for the University of Wollongong. For further information contact the UOW Library: research-pubs@uow.edu.au 


\title{
Sequential processing in the equiprobable auditory Go/NoGo task: children vs. adults
}

\begin{abstract}
Objective To compare sequential processing in the unwarned auditory equiprobable Go/NoGo task in children and adults, in the context of a recently developed adult schema. Methods Adult and child samples completed an equiprobable auditory Go/NoGo task while EEG was recorded from 19 channels. Go and NoGo ERPs were decomposed using unrestricted Varimax-rotated PCAs for the groups separately, and in combination. The separate adult and child components were compared using the Congruence Coefficient. Brain sources of each assessed component were examined using eLORETA. Results Corresponding adult/child components were tentatively identified: two N1 subcomponents (N1-1, PN) and P2, followed by N2, P3 (separate P3a/P3b in children), the classic Slow Wave (SW), and a diffuse Late Positivity (LP). While early and late components showed similarities, the intermediate P2 and N2 differed substantially in their stimulus effects. Conclusions Aspects of "Go" vs. "NoGo" categorisation differ between adults and children, but subsequent processing reflected in the different Go/NoGo P3 components, and their sequellae, are similar. Significance This is the first detailed examination of child responses in this paradigm. The tested schema appears relatively robust in adults, and the child results may aid our understanding of developmental aspects of cognitive processing in normal and atypical individuals.
\end{abstract}

\section{Keywords}

children, task, nogo, adults, go, vs, auditory, equiprobable, processing, sequential

\section{Disciplines}

Education | Social and Behavioral Sciences

\section{Publication Details}

Barry, R. J., De Blasio, F. M. \& Borchard, J. P. (2014). Sequential processing in the equiprobable auditory Go/NoGo task: children vs. adults. Clinical Neurophysiology, 125 (10), 1995-2006. 
Sequential Processing in the Equiprobable Auditory Go/NoGo Task: Children vs. Adults

Robert J. Barry*, Frances M. De Blasio, Jay P. Borchard

Centre for Psychophysics, Psychophysiology, and Psychopharmacology, Brain \& Behaviour

Research Institute, and School of Psychology, University of Wollongong, Wollongong 2522, Australia

*Corresponding author

Phone/fax: +6124221 4421

Email: robert_barry@uow.edu.au (R.J. Barry)

Address: School of Psychology, University of Wollongong, Wollongong NSW 2522, Australia.

Keywords: Children; Adults; ERPs; Equiprobable Go/NoGo paradigm; Principal Components Analysis (PCA); Exact Low Resolution Electromagnetic Tomography (eLORETA) 


\begin{abstract}
Objective: To compare sequential processing in the unwarned auditory equiprobable Go/NoGo task in children and adults, in the context of a recently developed adult schema. Methods: Adult and child samples completed an equiprobable auditory Go/NoGo task while EEG was recorded from 19 channels. Go and NoGo ERPs were decomposed using unrestricted Varimax-rotated PCAs for the groups separately, and in combination. The separate adult and child components were compared using the Congruence Coefficient. Brain sources of each assessed component were examined using eLORETA.

Results: Corresponding adult/child components were tentatively identified: two N1 subcomponents (N1-1, PN) and P2, followed by N2, P3 (separate P3a/P3b in children), the classic Slow Wave (SW), and a diffuse Late Positivity (LP). While early and late components showed similarities, the intermediate P2 and N2 differed substantially in their stimulus effects.

Conclusions: Aspects of “Go” versus “NoGo” categorisation differ between adults and children, but subsequent processing reflected in the different Go/NoGo P3 components, and their sequellae, are similar.

Significance: This is the first detailed examination of child responses in this paradigm. The tested schema appears relatively robust in adults, and the child results may aid our understanding of developmental aspects of cognitive processing in normal and atypical individuals.
\end{abstract}




\section{Highlights}

- Some early and late ERP components show similarities, but Go/NoGo P2 and N2 effects differ with age.

- This indicates that aspects of stimulus categorisation differ between children and adults.

- Subsequent processing reflected in P3 and later components is similar. 


\section{Introduction}

The equiprobable auditory Go/NoGo task, sometimes called a 50 \% auditory oddball task (Barry et al., 2000), is at the mid-point between traditional Go/NoGo tasks (with Go probability > NoGo probability) and the traditional oddball task (with Target probability < NonTarget probability). It generates ERPs that share features of the auditory oddball: sequential P1, N1, P2, N2, and P3 components, followed by the posterior-positive/anteriornegative classic Slow Wave (SW). Overall, the equiprobable Go ERPs appear similar to reduced oddball target ERPs, and NoGo ERPs appear similar to enhanced oddball standard ERPs (Duncan-Johnson and Donchin, 1977; Johnson, 1986), respectively. These ERPs also resemble in morphology those of the traditional Go/NoGo task, although as expected, the substantial NoGo N2 thought to represent inhibition or response conflict (Smith et al., 2013), is less pronounced in the equiprobable task. As in many other Go/NoGo tasks, as well as the oddball, P3 to the Go/target is larger and more parietal than that to the NoGo/standard. We follow Barry and Rushby (2006) who identified these P3 sub-components in this paradigm as P3b and P3a, respectively, as is generally compatible with the wider literature (e.g., Dien et al., 2004; Polich, 2007).

We have been interested in the unwarned equiprobable auditory Go/NoGo task for a number of years; specifically, it is the unique feature of equal stimulus presentations involving two very different processing chains that interests us. We have utilised this paradigm in brain dynamics studies exploring the genesis of the different ERP profiles for “Go" versus "NoGo” (e.g., Barry, 2009); and to examine the impact of prestimulus EEG on the subsequent ERP components, exploring the effects of phase (e.g., in children: Barry and De Blasio, 2012) and amplitude (e.g., in adults: De Blasio and Barry, 2013). However, our investigations were limited by the paucity of paradigm-specific processing information in the literature. In general, the expectation in this paradigm is a chain of broadly similar 
components to both Go and NoGo stimuli, with Go vs. NoGo effects anticipated in the N2 (anterior control-related NoGo N2 [Huster et al., 2013] vs. a more posteriorly negative Go N2 [Folstein and van Petten, 2008]), P3 (anterior NoGo P3a vs. posterior Go P3b [Rushby and Barry, 2006]), and SW components.

In order to clarify the processing chains involved in this paradigm in adults, we recently employed Principal Components Analysis (PCA) to assess the full range of ERP components associated with this task, particularly in regard to the differential (Go vs. NoGo) processing involved (Barry and De Blasio, 2013). In the adult sample, we found evidence of what were identified as an early P1 and N1-3 (Component 3 of the N1; Näätänen and Picton, 1987); these were not assessed due to their small variance. Following these sequentially we identified the N1-1 (Component 1 of the N1; Näätänen and Picton, 1987), Processing Negativity (PN; Näätänen and Picton, 1987), P2, N2, P3, classic SW, and a novel component we labelled as the "Late Positivity” (LP). Interestingly, Go vs. NoGo differences were found as early as the N1-1. The differential Go vs. NoGo pattern of results prompted the following interpretation of the processing stages and their indicators in this paradigm: N1-1 and PN mark the start of the identification of the characteristics defining Go/NoGo, and further sensory processing is reflected in the P2. Categorisation of the stimulus as "NoGo" results in a frontal N2, fronto-central P3, and an enhanced LP, while categorisation as "Go" is associated with a posterior N2 and P3, and classic SW, representing directed processing related to response preparation and execution. Our interest here was to investigate whether this response pattern could be replicated in an adult sample, and to explore the generality of this processing schema in the developmental context, assessing if it can also be found in children.

Specific information on child ERPs in the unwarned equiprobable auditory Go/NoGo task is minimal. In a study of prestimulus EEG phase effects on child ERP peak amplitudes 
using this paradigm, Barry and De Blasio (2012) reported a large frontocentral P1, frontocentral N1, centroparietal P2, frontocentral N2, and a P3 that was parietal to Go and central to NoGo. The early components were embedded in a large frontal negativity, similar to that found in children by Holcomb et al. (1986) using auditory paradigms. They had reported a large early broad negativity (100-300 ms) to targets and non-targets in an oddball task, that appeared to overlap N1, P2 and N2 components, and identified a late frontal negativity (350-700 ms) as the Nc common in children (Courchesne, 1977). These data are broadly compatible with child ERP morphology development reported for a 15\% auditory oddball (Johnstone et al., 1996), where the reduction in the early broad frontal negativity showed a linear trend from 8 to 17 years. A similar large early frontal negativity, centred on N2, was reported in 10-year olds in a Go/NoGo task with 30\% NoGo probability (Johnstone et al., 2005). The later N2 and P3 components were examined in 9-year olds by Jonkman et al. (2003) using a cued continuous performance task variant of the Go/NoGo task with $10 \%$ cued Go and cued NoGo trial pairs. They found elevated negativity in the N2 window for children, and this was greater for NoGo than Go. Jonkman et al. interpreted higher false alarm and impulsivity scores, together with the absence of a frontocentral NoGo P3 in children, in terms of a developmental lag in response inhibition. These data also complement the wider developmental literature. For instance, in a study of component amplitude changes from age 7 years to adulthood, Oades et al. (1997) reported developmental increases in N1 and P3, and developmental decreases in N2, together with maturational shifts towards adult topography. However, there are no relevant PCA studies in children.

The aims of this study were fourfold: (1) replicate the sequential processing schema in an adult sample; (2) explore the full range of child ERP components uncovered by the PCA in this paradigm; (3) infer the processing milestones in this paradigm in children; and (4) compare the processing chain between adults and children to provide insight into their 
developmental/processing differences. Previously we have used Low Resolution Electromagnetic Tomography (LORETA; Pascual-Marqui et al. 1994; Pascual-Marqui, 1999) to determine brain sources for adult P3a and P3b responses from 15 Go and 15 NoGo trials in a comparable auditory paradigm (Barry and Rushby, 2006), and were interested in identifying the sources of these and other components in both adults and children. Here we employed eLORETA (Pascual-Marqui, 2007, 2009), as an adjunct to our usual topographic scalp analysis.

\section{Methods}

\subsection{Participants}

The adult group consisted of 18 healthy University of Wollongong students (9 females, 9 males; 17 right-handed) recruited from the School of Psychology. Their mean age was 20.7 (range 18 - 30) years. The child group consisted of 18 healthy children (9 females, 9 males; 11 right-handed) recruited from the local region via newspaper advertisements. Their mean age was 10.3 (range 9 - 11) years. Subjects were screened for neurological disorders, head injury, learning disability and psychiatric conditions. All participants were required to abstain from caffeine and other psychoactive substances for at least $4 \mathrm{~h}$ prior to the testing session. Participation was voluntary and informed consent was obtained from the volunteer (adult sample), or parent/guardian (child sample), in line with a protocol approved by the joint University of Wollongong/South East Sydney and Illawarra Area Health Service Human Research Ethics Committee.

\subsection{Physiological recording}

Continuous EEG was recorded from 19 scalp sites ( $\times 20,000$ gain), using an electrode cap referenced to linked ears; care was taken to balance ear impedances. Vertical and horizontal electro-oculograms (EOGs) were also recorded (× 5,000 gain). Tin electrodes 
were used for both EEG and EOG recordings, and all impedances were below $5 \mathrm{~K} \Omega$. Data from 0.03 to $35 \mathrm{~Hz}$ were sampled by a 16 bit A/D system (AMLAB II) at $512 \mathrm{~Hz}$, and recorded for later off-line analysis.

\subsection{Task and procedure}

An unwarned equiprobable auditory Go/NoGo task was used. Stimuli were presented in blocks of 150 tones (50 ms duration, $5 \mathrm{~ms}$ rise/fall times), binaurally via headphones at 60 dB SPL, with a fixed stimulus onset asynchrony of 1100 ms. Half the tones were $1000 \mathrm{~Hz}$, and half $1500 \mathrm{~Hz}$, and these were presented in random order to avoid any consistent sequence effects between subjects. Adult participants received two stimulus blocks and, in anticipation of the greater loss of trials common in child recordings through increased artefact and lower performance levels, children received three blocks. Participants were instructed to press a button with their dominant hand in response to one of the tones, which was designated as the 'target'. The pitch of the 'target' tone $(1000$ or $1500 \mathrm{~Hz})$ was alternated between subjects.

\subsection{ERP quantification and analysis}

Waveforms were filtered (0.1 to $25 \mathrm{~Hz}$, zero-phase shift, $24 \mathrm{~dB} /$ Octave) and epoched offline using Neuroscan software (Compumedics, v. 4.3). Single trials containing muscular or other artefact, or incorrect responses (NoGo commissions; Go omissions or RTs $>500$ ms [adults] or $600 \mathrm{~ms}$ [children]) were excluded. ERPs were derived from -100 to $+750 \mathrm{~ms}$ relative to stimulus onset, and baselined relative to their prestimulus period.

Pre- and post-stimulus data (-100 ms to +750 ms: 436 data points) from 19 scalp locations were submitted to a PCA using Dien’s ERP PCA toolkit (v. 2.23; Dien, 2010) in MATLAB ${ }^{\circledR}$ (The Mathworks, v. 7.13, R2011b). Because we expected latency differences between our adult and child samples (which may strongly impact PCA stability), separate PCAs were initially conducted for the adult and child samples. Each included 36 data files (684 cases: 18 participants $\times 2$ conditions $\times 19$ sites). Data for the PCAs were half sampled 
to 218 time-points (variables) to reduce computation time and improve the case/component ratio to $\sim 3$. A third combined PCA included all subjects (72 data files, 1368 cases: 2 groups $\times 18$ participants $\times 2$ conditions $\times 19$ sites); this was used for clarity if similar components were obtained in the separate adult and child PCAs.

There is some disagreement in the PCA literature as to factor selection and rotation procedures, but there is recent recognition that the situation is relatively open (Dien, 2012). Hence our PCAs used the covariance matrix with Kaiser normalization, and all 218 unrestricted factors were subject to Varimax rotation, in line with recommendations published in this journal (Kayser and Tenke, 2003). The waveform of each extracted component was calculated by multiplying the factor loadings by the factor scores, and then rescaling the product to microvolts (by multiplying by the standard deviations of each timepoint in the original data). Conveniently, the ERP PCA toolkit (Dien, 2010) delivers this computation for each component, plotting the time-course of the component waveforms at the site of maximal component amplitude, beside their corresponding topographic headmaps, representing the component amplitudes (across the scalp sites) at the peak component latency. We examined these plots when identifying PCA factors as ERP components; starting with the factors that account for the largest proportion of the variance in the data, the latency, polarity, and topographic distribution of the components informed our selections. We also considered the latency and topography of the peaks apparent in the raw ERPs, and their correspondence with those of the components thought to approximate them. Finally, the sum of the waveforms of the identified ERP components (i.e., the 'reconstituted ERP') was compared with the original ERP to visually assess how well the combination of these factors approximated the raw data.

In order to formally assess the similarity of components extracted from the separate adult and child sample PCAs, the Congruence Coefficient (Tucker, 1951) was computed for 
each pair of corresponding components. This is a form of Pearson's correlation of the factor loadings over time, using variables without the usual mean correction. In essence, it compares the temporal characteristics of components, and is reduced by differences in peak latency, onset/offset times, and relative magnitudes. Similarity of components between two data sets can be assessed using a rule-of-thumb, with $r_{c}>.95$ being taken to indicate equality of components, and $r_{c}<.85$ indicating dissimilarity (Lorenzo-Seva and ten Berge, 2006). As factor loadings do not carry topographic information, the Congruence Coefficient is not sensitive to component topographic similarities or differences.

To allow comparison of topography and Go/NoGo effects, separate repeatedmeasures MANOVAs were conducted for the adult and child samples. These examined the effects of Condition (Go vs. NoGo) for each of the identified components, assessing the component amplitudes at 9 core sites (F3, Fz, F4, C3, Cz, C4, P3, Pz, P4). Each analysis included examination of topography, with sagittal plane (frontal [F3, Fz, F4], central [C3, Cz, C4] and parietal [P3, Pz, P4]) and coronal plane (left [F3, C3, P3], midline [Fz, Cz, Pz] and right $[\mathrm{F} 4, \mathrm{C} 4, \mathrm{P} 4])$ as repeated-measures factors. Planned contrasts within the sagittal plane compared frontal vs. parietal regions, and their mean vs. central sites. Within the coronal plane, the left vs. right regions, and their mean vs. the midline sites, were analysed. These orthogonal planned contrasts and their interactions provide optimal information on the topographic distribution of the amplitude of each component, completely specifying the nine regional relativities. Since all contrasts were planned and there were no more of them than the degrees of freedom for effect, no Bonferroni-type adjustment to $\alpha$ was necessary (Tabachnick and Fidell, 1989). Also, Greenhouse-Geisser type correction was not necessary because single degree of freedom contrasts are not affected by the violations of sphericity assumptions common in repeated-measures analyses of physiological data (O’Brien and Kaiser, 1985). All $F$ tests reported have $(1,17)$ degrees of freedom unless otherwise 
specified. In addition to significant effects $(p<.05)$, those at $.10>p \geq .05$ are reported to encourage further research, but are not discussed.

Finally, the sources of each of the selected components to Go and NoGo stimuli in each group were examined using eLORETA (Pascual-Marqui, 2007, 2009). This simple examination of sources was based on grand mean components as a complement to the statistical analyses of their scalp topography.

\section{Results}

As expected, Table 1 shows that the adult group was significantly older, and had significantly reduced mean RT and proportion of errors of both omission (failure to buttonpress to Go) and commission (button-press to NoGo) than the child sample. Because of the difference in the number of trial blocks presented to the adults (two) and children (three), both groups had comparable numbers of Go and NoGo trials available for ERP averaging after rejection of trials containing errors and artefacts.

\section{Table 1 about here.}

\subsection{Grand mean ERPs}

Midline grand mean ERPs are shown in Figure 1 (left column), and the prominent expected components are indicated at Fz. In adults (solid traces), a small P1 is followed by a marked frontocentral N1 ( 100 ms) and a prominent P3 ( 300 ms); Go P3 (black) is posterior, and NoGo P3 (grey) is central. P3 appears to be followed by a classic frontalnegative/posterior-positive SW around $420 \mathrm{~ms}$, with the subsequent LP that appears more positive for NoGo (grey). P2 and N2 peaks are apparent as inflection points between the N1 and P3 peaks; these are small relative to the dominant components. In children (dashed traces), similar component peaks are apparent at similar latencies. P1, N1, P2 and N2 are quite marked, particularly at $\mathrm{Cz}$, but P3 is relatively small. There is also a broad enhanced 
early frontal negativity apparent from $100 \mathrm{~ms}$ in the child ERPs; this causes the P2 (apparent trough between N1 and N2) and P3 (apparent trough following N2) to appear negative at $\mathrm{Fz}$ (c.f. the positivity of the corresponding peaks at $\mathrm{Cz}$ and $\mathrm{Pz}$ ). There was also some indication of a frontal-negative/posterior-positive SW, and LP.

Figure 1 about here.

\subsection{Separate PCA outcomes}

Of the 218 rotated components in adults, the first seven were identified in terms of their sequence, polarity, latency, topography, and similarity to peaks in the raw ERPs and to previous PCA outcomes in this paradigm (Barry and De Blasio, 2013). These are the N1-1, PN, P2, N2, P3, the classic SW, and LP. Two additional identifiable components are the early P1 and N1-3; these components (factors 10-11) followed, in factor order, a small unidentifiable frontal negativity at $564 \mathrm{~ms}$ and a small unidentifiable temporal positivity at 416 ms. Note that, of the identified components, only the subcomponents of the N1 cannot be separately identified in the raw ERPs of Figure 1 (left column, solid traces). Together the nine identifiable components explained 93.2\% of the variance. The reconstituted adult ERP, being the sum of the nine identified adult components, is displayed for each of the midline sites in Figure 1 (right column, solid traces); comparison with the mean ERPs (left column, solid traces) confirms a good approximation of the original data.

For the child data, the first ten of the 218 rotated factors were identifiable in terms of their broad similarity to the adult components, in addition to their sequence and latency, and to a lesser extent, their polarity and topographic distribution. A notable difference was an increase in the frontal negativity of the child (c.f. adult) components within the 150-300 ms latency range, likely reflecting the difference in the mean ERPs (Figure 1, left column, dashed vs. solid traces), and the appearance of two small P3 components rather than the single large adult component. In latency order, the components were tentatively identified as 
P1, N1-3, N1-1, PN, P2, N2, P3a, P3b, SW, and LP. Again, note that only the subcomponents of the N1 are not separable in the raw ERPs (Figure 1, left column, dashed traces), as there is some suggestion of two P3s near 300 and 400 ms. These components explained 93.8\% of the variance, and the sum of their waveforms, the reconstituted child ERP, is displayed at each of the midline sites in Figure 1 (right column, dashed traces); comparison with the mean ERPs (left column, dashed traces) again confirms a good fit with the original data.

For the adult PCA, Figure 2 (upper panel) displays the loadings of the factors plotted against time; these represent the unscaled correlations between the component and the ERP waveform (Tabachnick and Fidell, 1989). Below this, the latency, percentage of the total variance accounted for, and factor number are indicated above the corresponding topographic headmap for each of the components, averaged across condition. The child PCA data are presented in the lower panel of Figure 2; the topographic headmaps of the identified components averaged across condition appear above their corresponding factor number, percentage of the total variance accounted for, and latency. Below these are the loadings of the factors displayed against time. Note that each headmap is plotted with a scale of $\pm 7 \mu \mathrm{V}$; although the topographies of the weaker components (i.e., adult N2, child P1) become somewhat harder to evaluate at this scale, the magnitude of the component amplitudes are emphasised (c.f. the prominent components of the mean ERPs in Figure 1 with their respective component headmaps in Figure 2). Because of space restrictions, the early P1 and N1-3 are not discussed further.

Figure 2 about here.

\subsection{Temporal comparison of separate adult/child PCA components}

The Congruence Coefficients $\left(r_{c}\right)$ for the adult c.f. child PCA factor loadings are shown between the component label pairs in the centre line of Figure 2. These were 
evaluated using the common rule-of-thumb for interpreting this coefficient (Lorenzo-Seva and ten Berge 2006). N1-1 was approx. 8 ms later in children than in adults, and the temporal PN was approx. 4 ms later; each of these N1 subcomponents showed 'fair similarity' between the adult/child samples $\left(.85<r_{c}<.94\right)$. Although P2 was only some 4 ms earlier in children than in adults, and the child N2 peaked some 11 ms later than the adult $\mathrm{N} 2$, neither showed 'similarity' between the adult and child samples $\left(r_{c}<.85\right)$. These dissimilarities were not unexpected given the clear differences seen in Figure 2 in the factor loading plots (P2: adult factor 5 vs. child factor 7; N2: adult factor 7 vs. child factor 1), variance accounted for, polarity, and topographic distributions between the adult and child components. For P3, the adult factor loading was compared with the sum of the child P3a and P3b factor loadings. The two child P3s were approx. 12 and 59 ms later than the single adult P3, and their composite had 'no similarity' $\left(r_{c}<.85\right)$ to the adult P3; again this was not surprising given the substantial adult/child P3 component differences evident in Figure 2. Despite the child SW being approx. $31 \mathrm{~ms}$ later, and the LP some $8 \mathrm{~ms}$ later than in adults, the corresponding congruence coefficients indicated that these components can be considered identical $\left(r_{c}>.95\right)$ in adults and children.

These results suggested that the ‘similar' early (N1-1, PN) and 'equal’ late (SW, LP) components should be examined in the combined PCA, which included all samples, adult and child. In contrast, the intermediate components (P2, N2, P3) differed between the samples, so we continued to assess these sample-specific components as uniquely expressed in the separate adult and child PCAs.

\subsection{Combined PCA}

The first 10 factors of the combined adult/child PCA accounted for $>1 \%$ of the variance individually and $94.0 \%$ of the variance together. The loadings of the factors plotted against time are displayed at the top in Figure 3. Below this, the component labels (for 
identified components), latency, percentage of the total variance accounted for, and factor number, are indicated above the corresponding topographic headmap for each component for each group, averaged across condition.

Figure 3 about here

The early and late identified components (labelled) show latency and topography similar to those from the separate PCAs (Figure 2). The combined Factor 1 appears to be an unacceptable amalgam of the separate child N2 and adult P3, and there is no convincing P2. Other components reflect aspects of the separate adult/child components, but it does not appear profitable to pursue these further, so no effort has been devoted to their identification and labelling.

\subsection{PCA component topographies and amplitudes}

Here we examine the N1-1, PN, SW and LP from the combined PCA, and the intermediate P2, N2, P3 components from the separate PCAs. Relative to the corresponding adult components, Figure 3 indicates that the child N1-1 was weaker overall, and more dominant in the right frontal region, and the temporal PN was stronger and associated with a vertex positivity. As shown in Figure 2, the children's P2 lacked a vertex positivity, their frontal N2 was very dominant compared with the adult N2, and the two child P3s were much smaller than the single adult P3. The child SW in Figure 3 was relatively more positive, while the LP appeared weaker and more parietal than in adults.

The separate Go and NoGo topographic headmaps from the relevant PCAs are shown for each group in Figure 4; the adult data are presented in the upper panel, and the child data in the lower panel. Statistical results of the topographic and Go/NoGo analyses are presented in Tables 2 and 3, respectively. To aid in comparison of the results, the adult data are presented in the left column, and the child data are presented in the right. Underlined statistical results indicate a reversal of the corresponding underlined contrast for that effect or 
interaction; for example, in Table 2, the first effect in $\mathrm{P} 3$ indicates that $\mathrm{F}<\mathrm{P}$ applies to the adult P3 ( $F$ value not underlined) but the effect is reversed for the separate child P3a ( $F$ value underlined). Significant results are indicated by a bold $p$ value.

Figure 4 about here. Tables 2 \& 3 about here.

\subsection{1. $N 1-1$}

Across conditions, the adult N1-1 was frontocentral, as seen in Figure 3, and the frontal distribution was increased somewhat in the hemispheres, relatively more so in the right. N1-1 to Go (c.f. NoGo) was enhanced parietally, and somewhat in the midline. Overall Go N1-1 was greater than NoGo N1-1; compare adult Go vs. NoGo N1-1 headmaps in Figure 4.

Across conditions, the child N1-1 was frontal and midline dominant. The midline (c.f. hemispheric) enhancement was larger parietally, an interaction statistically equivalent to the frontal hemispheric dominance noted in adults. There was also some right hemisphere enhancement, and this increased somewhat centrally; see Figure 3. There was no effect of Go/NoGo in the child N1-1.

\subsection{2. $P N$}

Across Go and NoGo, and over the nine core scalp sites initially analysed, the adult PN was enhanced in the frontal right hemisphere; this can be seen in Figure 3. Go PN was reduced frontally, particularly at the midline; and was also reduced at the vertex. Go PN was larger in the hemispheres, particularly in the left hemisphere; see Figure 4. Over the 9 core sites, NoGo PN was more negative than Go PN.

Across Go and NoGo, and over the nine core scalp sites initially analysed, the PN in children was frontal with a strongly reduced central negativity, and was greater in the hemispheres than midline, together leading to a vertex minimum (i.e., positivity); this can be seen in Figure 3. Go PN was relatively larger parietally due to the parietal positivity in the 
NoGo PN; compare child Go/NoGo PN headmaps in Figure 4. Also, the vertex PN reduction was increased for Go stimuli. There was no main effect of condition.

In the adult PN, a supplementary analysis including the outer electrodes to reflect the temporal nature of the PN (i.e., replacing the F3/4, C3/4, P3/4 electrode pairs with F7/8, T3/4, T5/6) found a central elevation, and some frontal enhancement in the right hemisphere. Go PN was less negative frontally, and was increased in the hemispheres, particularly in the left hemisphere. The hemispheric enhancement was larger for Go PN in parietal and central regions; see Figure 4. Together these analyses indicate that the temporal PN was greater for Go than NoGo, despite an overall reduction in negativity.

The same supplementary analysis of the child PN found a relative elevation in the hemispheres, particularly in the right; the former was predominant in the central (temporal) regions; see Figure 3. There was some enhanced frontality in the right hemisphere that was significant in the midline. A frontal PN enhancement was somewhat smaller for Go, and the hemispheric enhancement in the temporal regions was somewhat larger for Go, indicating that the temporal PN was somewhat greater for Go than NoGo; see Figure 4.

\subsection{3. $P 2$}

As seen in Figure 2, P2 in the adults was central across conditions, with a midline dominance, and vertex maximum. NoGo P2 was enhanced centroparietally, and in the left central region; this is clearly evident in Figure 4.

Figure 2 shows that P2 in the children was parietal across conditions. Go P2 was enhanced (i.e., less negative), and the NoGo P2 reduced, in the midline, particularly centrally. Go P2 was also increased in the central left; this is shown in Figure 4.

\subsection{4. $N 2$}

Across conditions, Figure 2 shows that the adult N2 was dominant frontally with a central reduction; and enhanced in the hemispheres, particularly centrally, and somewhat 
frontally. As evident in Figure 4, N2 for Go was somewhat greater (i.e., more negative) centrally, and greater in the midline, particularly at the vertex.

Across conditions, Figure 2 shows that the child N2 was frontal, and somewhat greater in the hemispheres. The frontal N2 enhancement was greater in Go, and there was some indication of a central enhancement, which reached significance at the vertex, in comparison with the frontally dominant NoGo N2. These effects were due to the substantial parietal positivity in the Go N2 evident in Figure 4; hence overall, the N2 was significantly greater (i.e., more negative) for NoGo than Go.

\subsection{4. $P 3$}

As is apparent in Figure 2, the single adult P3 (over conditions) was centroparietal, and midline dominant with a left bias. The central enhancement was greatest in the midline, and the left bias was greater centroparietally. P3 was parietal for Go, and somewhat more central for NoGo; this can be seen in Figure 4. The left bias of P3 was enhanced in the Go response, particularly parietally. The central NoGo P3 enhancement was also larger on the left, and was largest at the vertex.

The first of the two P3 components in the children was labelled as the P3a. As is apparent in Figure 2, the child P3a (over conditions) was frontocentral, midline dominant, and the frontal enhancement was greatest in the midline. The central enhancement was greater for NoGo than Go; this can be seen in Figure 4 (lower panel). There was also some left bias of P3a in the NoGo response.

The second P3 component in the children was labelled as the P3b. As seen in Figure 2, across conditions the child P3b was midline dominant, particularly frontally. A parietal enhancement was dominant in the Go P3b, whereas a central enhancement was greater for NoGo; this can be seen in Figure 4. Moreover, the central enhancement in the NoGo P3b showed a left bias, and was largest at the vertex. 


\subsubsection{SW}

Across conditions, the adult SW was centroparietally positive and frontally negative; see Figure 3. The parietal positivity was larger on the left. In the midline, the frontal negativity/parietal positivity was enhanced, and the central positivity was reduced. As evident in Figure 4, the Go SW was enhanced centrally and in the left hemisphere, and somewhat so in the midline; and the defining frontal negativity/parietal positivity was enhanced, particularly in the left hemisphere, and somewhat in the midline. Overall, the SW was greater for Go than NoGo.

Across conditions, the child SW was centroparietally positive and frontally negative; see Figure 3. The SW was reduced in the midline, particularly the central positivity, although the frontal negativity/parietal positivity was enhanced here. As evident in Figure 4, the SW centroparietal positivity and frontal negativity of the child SW were both enhanced in the Go response; the enhancement in parietal positivity was greater in the midline, whereas the central positivity enhancement was greater in the hemispheres, particularly in the right. Overall, the SW was substantially greater for Go than NoGo.

\subsection{6. $L P$}

The adult LP was globally positive and larger in the right hemisphere, particularly centrally, and larger frontally in the hemispheres; see Figure 3. As clearly apparent in Figure 4, the adult NoGo LP was relatively uniform over the scalp, with the Go LP reduced in the left hemisphere, more so centrally; and somewhat smaller in the midline. Overall, the LP was enhanced for NoGo compared with Go.

The child LP was centroparietally positive and frontally negative, with a reduced midline; see Figure 3. In the midline, the parietal positivity and frontal negativity were enhanced, and the central positivity was reduced; also, the central positivity was greater on the right. As apparent in Figure 4, there was some indication of NoGo LP enhancements in 
the left frontocentral region; the NoGo response was significantly larger in the midline, and at the vertex, but was decreased across the central region. Overall, the LP was more uniform over the scalp and enhanced for NoGo.

\subsection{PCA component source localisation}

For illustration, we include eLORETA source plots for one component, arbitrarily chosen for its similarity between the groups: Figure 5 shows the combined PCA N1-1 Go (top) and NoGo (bottom) components in the adult (left) and child (right) samples;

Supplementary Figure S1 shows the corresponding plots for all components analysed. Table 4 lists the dominant eLORETA sources for each PCA component in each condition, with similar adult/child source localisations in each condition indicated in bold font.

Table 4 \& Figure 5 about here.

For the adult data, an initial scan through Table 4 shows some commonality of sources justifying the same component label for Go (first column) and NoGo (third column) responses for N1-1 (Inferior Temporal Gyrus, Middle Temporal Gyrus), P2 (Postcentral Gyrus), P3 (Medial Frontal Gyrus), and LP (Medial Frontal Gyrus, Superior Frontal Gyrus). In comparison, the child Go (second column) and NoGo (fourth column) data show a different source commonality for N1-1 (Superior Temporal Gyrus, Postcentral Gyrus), PN (Precentral Gyrus), N2 (Postcentral Gyrus), and P3a (Superior Frontal Gyrus, Medial Frontal Gyrus). Adult components: PN, N2, and SW; and Child components: P2, P3b, SW and LP, did not share commonalities; see Supplementary Figure S1.

Similar sources (bolded) for adult/child Go responses are apparent in Table 4 (first and second columns) for N1-1 (Superior Temporal Gyrus; also see Figure 5), P3 when compared with P3a (Medial Frontal Gyrus) and P3b (Inferior Frontal Gyrus), SW (Medial Frontal Gyrus), and LP (Middle Frontal Gyrus); but not for PN, P2 or N2. For NoGo responses (third and fourth columns), similar adult and child sources are apparent for N2 
(Postcentral Gyrus) and the adult P3 and child P3a (Medial Frontal Gyrus), but not for N1-1, PN, P2, P3b, SW, or LP; see Supplementary Figure S1.

\section{Discussion}

This is our second investigation assessing the PCA components of the unwarned equiprobable auditory Go/NoGo task with the aim of mapping the sequential processing milestones involved in each condition, and the first to compare adult vs. child processing. The adult ERPs here demonstrated results similar to those found in our previous study (Barry and De Blasio, 2013). The adult N1-1 and temporal PN showed substantial enhancements to Go, supporting the interpretation of differential processing beginning at this early stage. P2 was enhanced to NoGo, and N2 was enhanced at the vertex in the Go condition. The parietal P3b was apparent in response to Go stimuli, while the central P3a was enhanced in response to NoGo. Finally, the SW was enhanced to Go, and LP was greater to NoGo stimuli.

Note that these component labels reflect those used in Barry and De Blasio (2013), and some of these could be questioned. For example, it was suggested in the review process for this paper that our component labelled "PN" - at least in children, where it has a vertex positivity - closely matched the temporal-N1 or T-complex of Näätänen and Picton (1987), as reported in adults by Kayser and Tenke (2006) and Tenke and Kayser (2012), and should be so labelled. However, Näätänen and Picton (1987) described the positivity of the Tcomplex at $100 \mathrm{~ms}$, well before its negativity at $150 \mathrm{~ms}$, a marked discrepancy from the 150 ms positivity and negativity apparent in our children and in the Kayser and Tenke data. Hence we retain the PN label for our adults, and propose its tentative use for the $154 \mathrm{~ms}$ component observed in children. Also, the LP was a novel finding of Barry and De Blasio (2013) and it could be queried as merely an outcome of the autocorrelation of EEG time series at the end of the baselined ERP epoch (Kayser and Tenke, 2003). However, we 
consider this to be a genuine component as it peaks some $100 \mathrm{~ms}$ prior to the end of the present PCA epoch (Figure 1), and a follow-up extension of the ERP epoch indicated substantial resolution of the Go/NoGo difference around $800 \mathrm{~ms}$ in adults, and by $900 \mathrm{~ms}$ in children. Further discussion of this issue is beyond the present study, but it should be investigated further in future work.

\subsection{Sequential processing in adults}

The present results can be taken as supporting our proposed Go/NoGo processing schema in adults (Barry and De Blasio, 2013). That is, our adult data support the following: at stimulus onset, initial stimulus transient processing is reflected in the N1-1, and further sensory processing is reflected in the PN and P2. Go responses are larger than NoGo (topographically or globally) in N1-1 and the temporal PN, indicating that identification of the stimulus characteristics defining Go/NoGo begins at these early processing stages; this is confirmed by the larger NoGo P2. We equate the NoGo P2 with Oades et al.’s (1996) suggestion that this inhibition clears the way for processing of the target Go stimulus, compatible with it modulating attention to facilitate stimulus discrimination (Crowley and Colrain, 2004). Subsequent processing to complete categorisation of the stimulus is then reflected in the N2. If the outcome of categorisation is "NoGo", only basic processing continues, resulting in the central NoGo P3a, followed by an enhanced LP; this diffuse positivity indicates a broad cortical deactivation marking the end of active stimulus processing (Barry and De Blasio, 2013). Conversely, if the stimulus is categorised as "Go", there is a surge in directed, effortful processing, response preparation and execution, reflected in the vertex N2 activity, maximal parietal Go P3b, and a large frontally negative/centroparietally positive Go SW.

\subsection{Adult vs. child PCA outcomes}

The child data demonstrated ERPs broadly similar to those found in our previous 
child study in this paradigm (Barry and De Blasio, 2012), to our developmental work with the auditory oddball (Johnstone et al., 1996) and non-equiprobable auditory Go/NoGo (Johnstone et al., 2005) tasks, to Holcomb et al. (1986), and to Oades et al. (1996, 1997), being particularly characterised by a large frontal N2 and smaller P3 (see dashed traces, left column of Figure 1). The separate child PCA generated a range of components that were similar to the adult data in some respects, and different in others, in line with the apparent adult vs. child ERP differences observed in Figure 1 (left column).

One surprising novel observation that deserves attention emerges from detailed consideration of the topographies in Figure 3. Compared with adults, many of the child components display marked positive temporal activity (particularly N1-1, P3b, and the SW). We conducted supplementary analyses of the temporal activity in the PN based on our previous work, but did not do so here for these unexpected observations. This enhanced temporal positivity in many components should be examined in future child studies.

The child N1 subcomponents (N1-1 and PN) had loadings very similar to the corresponding subcomponents in adults, allowing their joint extraction in the combined PCA. In contrast to these components in adults, the child N1-1 appeared more right frontal in topography and was somewhat weaker, and the PN was stronger in both its temporal negativity and vertex positivity (see Figure 4). In terms of Condition effects, the child N1-1 did not differ between Go and NoGo, but the PN was enhanced to Go stimuli, similarly to the adult N1-1, suggesting differential Go/NoGo processing may begin later in children, but is apparent from PN onwards.

The coefficient of congruence for P2 suggested no similarity between the child and adult components, consistent with the finding that the child P2 was enhanced to Go, while in adults it was larger to NoGo. This suggests that, unlike in adults, the child P2 does not reflect inhibition of the NoGo stimulus from further processing. However, further work is required 
to investigate the role of this component in children in this paradigm.

The components identified as N2 in children and adults were also found to have a small coefficient of congruence indicating substantial component dissimilarity. The child N2 displayed a strong frontal topography and was greater overall for NoGo, while the N2 in adults was relatively weak frontally and was instead enhanced at the vertex for Go responses (compare N2 headmaps, Figure 4).

The child/adult differences in the topography and functionality of P2 and N2 are worth investigating in future studies. Although these differences have been found here in the unwarned equiprobable auditory Go/NoGo task, it is unlikely that they are unique to this paradigm. Indeed, topographic peak differences in P2 and N2 have been long reported (e.g., from our own laboratory: Johnstone et al., 1996, 2005). Given the well-established association between N2 and inhibition, it would be interesting to see if there would be any indication of PCA N2 component similarity between child and adult samples with the use of a paradigm that would elicit this process more directly, such as a traditional Go/NoGo task (e.g.., with $80 \%$ Go probability).

The combined P3s in children were found to have a low coefficient of congruence in relation to the adult component, and thus could be expected to have no similarity. However, although P3 was relatively weak in children compared with adults, and emerged in two separate components, both groups had a more central P3a in response to NoGo, and a parietal P3b in response to Go, suggesting similar differential stimulus processing across the age groups.

The coefficients of congruence for the SW and LP were very high, suggesting equivalent components in the child and adult samples. Components from the combined PCA confirmed this: in both groups, the SW was enhanced to Go, and the LP was greater to NoGo stimuli, suggesting that these components serve similar functions in child and adult 
processing.

\subsection{Source localisation}

We carried out a simple eLORETA examination of the major sources involved in the assessed PCA Go/NoGo components obtained from our adult and child samples, based on the separate topographic means for each group. The obtained sources are broadly comparable with previous adult data from the oddball task (e.g., Volpe et al., 2007; Saletu et al., 2008) and the traditional Go/NoGo task (e.g., Bokura et al., 2001). Barry and Rushby (2006) reported major LORETA P3 sources in the cuneus, cingulate gyrus, and precuneus for Go, and the cingulate gyrus, cuneus and anterior cingulate for NoGo; only partially overlapping with the present findings for the child Go P3a (cingulate gyrus), and the adult NoGo P3 (anterior cingulate). Some of these localisation discrepancies may be attributed to the computational differences between LORETA versions. There are no other relevant LORETA data from the unwarned equiprobable auditory Go/NoGo task in adults or children.

Obviously, much more research is necessary to replicate and extend these data, but the results here provide a starting point for investigations in this paradigm. The present mass of novel data in Table 4 and Supplementary Figure S1 may provide a foundation to encourage future exploration in this area.

\subsection{Sequential processing in children}

In the light of these adult/child differences in component timing, topography, stimulus relations, and source localisations, it is very obvious that we can at present make no claim of identity between adult/child ERP components identified here with the same label. These labels should be regarded as a shorthand device to facilitate communication, rather than always using (say) “adult-N1-1” vs. “child-N1-1”, or as hypotheses requiring testing and development in further research.

With this limitation in mind, the present results allow us to tentatively extend our 
Go/NoGo processing schema (Barry and De Blasio, 2013) to children. That is, at stimulus onset, initial stimulus transient processing is reflected in the N1-1, and further sensory processing is reflected in the PN and P2. PN (but not N1-1) is enhanced to Go stimuli in both groups, indicating that this stage of identification of the stimulus characteristics defining Go/NoGo is progressing similarly for adults and children.

Subsequently, different adult/child processing is evidenced by the larger Go P2 and NoGo N2 in children, versus the larger NoGo P2 and Go N2 in adults; this suggests that stimulus processing and categorisation in children may persist beyond that in adults. Interestingly, although P3 was relatively weak in children compared with adults (with a coefficient of congruence suggesting no similarity), and emerged in two separate subcomponents, both groups showed the NoGo P3a/Go P3b topographic separation typical in this paradigm (Barry and Rushby, 2006). This suggests that the immature P3 subcomponents function similarly to their adult counterparts. Subsequently, the dominant Go SW and NoGo LP appeared to be virtually identical in adults and children, and showed corresponding processing-related patterns in each group. Thus, after stimulus categorisation, our previously-suggested sequential processing schema is apparent in both adults and children. If the outcome of categorisation is "NoGo", simple "basic" processing continues, resulting in the central NoGo P3a, followed by an enhanced LP indicating that the active stimulus processing has ceased (Barry and De Blasio, 2013). If the stimulus is categorised as "Go", there is a surge in directed, effortful processing with a maximal parietal Go P3b, followed by a large frontally negative/centroparietally positive Go SW.

These child/adult similarities and differences in the sequential processing necessary for adequate functioning in the unwarned equiprobable Go/NoGo task need further exploration to help us understand their development over the lifespan. It would be interesting to explore our proposed processing schema in child groups of different ages to see if there is 
evidence of the differences noted above changing towards the adult profile with increasing age. It would also be interesting to see how our proposed schema holds for well-functioning elderly participants, and whether that might help provide insight into processing deficits associated in other elderly groups showing cognitive decline, and perhaps Alzheimer's dementia. 


\section{References}

Barry RJ. Evoked activity and EEG phase resetting in the genesis of auditory Go/NoGo ERPs. Biol Psychol 2009; 80:292-299.

Barry RJ, De Blasio FM. EEG-ERP phase dynamics of children in the auditory Go/NoGo task. Int J Psychophysiol 2012; 86:251-261.

Barry RJ, De Blasio FM. Sequential processing in the equiprobable auditory Go/NoGo task: A temporal PCA study. Int J Psychophysiol 2013; 89:123-127.

Barry RJ, Kirkaikul S, Hodder D. EEG alpha activity and the ERP to target stimuli in an auditory oddball paradigm. Int J Psychophysiol 2000; 39:39-50.

Barry RJ, Rushby JA. An orienting reflex perspective on anteriorisation of the P3 of the event-related potential. Exp Brain Res 2006; 173:539-545.

Bokura H, Yamaguchi S, Kobayashi S. Electrophysiological correlates for response inhibition in a Go/NoGo task. Clin Neurophysiol 2001; 112:2224:2232.

Courchesne E. Event-related brain potentials: Comparison between children and adults. Science 1977; 197:589-592.

Crowley KE, Colrain IM. A review of the evidence for P2 being an independent component process: Age, sleep and modality. Clin Neurophysiol 2004; 115:732-744.

De Blasio FM, Barry RJ. Prestimulus delta and theta determinants of ERP responses in the Go/NoGo task. Int J Psychophysiol 2013; 87:279-288.

Dien J. The ERP PCA Toolkit: An open source program for advanced statistical analysis of event-related potential data. J Neurosci Methods 2010; 187:138-145.

Dien, J. Applying Principal Components Analysis to event-related potentials: A tutorial. Dev. Neuropsychol 2012; 37: 497-517. 
Dien J, Spencer KM, Donchin E. Parsing the late positive complex: Mental chronometry and the ERP components that inhabit the neighborhood of the P300. Psychophysiol 2004; 41:665-678.

Duncan-Johnson CC, Donchin E. On quantifying surprise: The variation of event-related potentials with subjective probability. Psychophysiology 1977; 14:456-467.

Folstein JR, van Petten C. Influence of cognitive control and mismatch on the N2 component of the ERP: A review. Psychophysiology 2008; 45:152-170.

Holcomb PJ, Ackerman PT, Dykman RA. Auditory event-related potentials in attention and reading disabled boys. Int J Psychophysiol 1986; 3:263-273.

Huster RJ, Enriquez-Geppert S, Lavallee CF, Falkenstein M, Herrmann CS. Electroencephalography of response inhibition tasks: Functional networks and cognitive contributions. Int J Psychophysiol 2013; 87:217-233.

Johnson Jr R. A triarchic model of P300 amplitude. Psychophysiology 1986; 23:367-384.

Johnstone SJ, Barry RJ, Anderson J, Coyle SF. Age-related changes in child and adolescent event-related potential component morphology, amplitude and latency to standard and target stimuli in an auditory oddball task. Int J Psychophysiol 1996; 24:223-238.

Johnstone SJ, Pleffer CB, Barry RJ, Clarke AR, Smith JL. Development of inhibitory processing during the Go/NoGo task: A behavioural and event-related potential study of children and adults. J Psychophysiol 2005; 19:11-23.

Jonkman LM, Lansbergen M, Stauder JEA. Developmental differences in behavioral and event-related brain responses associated with response preparation and inhibition in a go/nogo task. Psychophysiol 2003; 40:752-761.

Kayser J, Tenke CE. Optimizing PCA methodology for ERP component identification and measurement: Theoretical rationale and empirical evaluation. Clin Neurophysiol 2003; 114:2307-2325. 
Kayser J, Tenke CE. Principal components analysis of Laplacian waveforms as a generic method for identifying ERP generator patterns: I. Evaluation with auditory oddball tasks. Clin Neurophysiol 2006; 117:348-368.

Lorenzo-Seva U, ten Berge JMF. Tucker’s congruence coefficient as a meaningful index of factor similarity. Methodology 2006; 2:57-64.

Näätänen R, Picton T. The N1 wave of the human electric and magnetic response to sound: A review and an analysis of the component structure. Psychophysiology 1987; 24:375425.

Oades RD, Dittmann-Balcar A, Schepker R, Eggers C, Zerbin, D. Auditory event-related potentials (ERPs) and mismatch negativity (MMN) in healthy children and those with attention-deficit or Tourette/tic symptoms. Biol Psychol 1996; 43:163-185.

Oades RD, Dittmann-Balcar A, Zerbin D. Development and topography of auditory eventrelated potentials (ERPs): Mismatch and processing negativity in individuals 8-22 years of age. Psychophysiology 1997; 34:677-693.

O’Brien RG, Kaiser MK. MANOVA method for analyzing repeated measures designs: An extensive primer. Psychol Bull 1985; 97:316-333.

Pascual-Marqui RD. Review of methods for solving the EEG inverse problem. Int J Bioelectromag 1999; 1:75-86.

Pascual-Marqui RD. Discrete, 3D distributed, linear imaging methods of electric neuronal activity. Part 1: Exact, zero error localization. arXiv:0710.3341 [math-ph] 2007.

Pascual-Marqui RD. Theory of the EEG inverse problem. In: Tong S, Thakor NV, editors. Quantitative EEG analysis: Methods and clinical applications. Boston: Artech House, 2009: 121-140. 
Pascual-Marqui RD, Michel CM, Lehmann D. Low resolution electromagnetic tomography: A new method for localizing electrical activity in the brain. Int J Psychophysiol 1994; 18:49-65.

Polich J. Updating P300: An integrative theory of P3a and P3b. Clin Neurophysiol 2007; 118:2128-2148.

Saletu M, Anderer P, Saletu-Zyhlarz GM, Mandl M, Zeitlhofer J, Saletu B. Event-related potential low-resolution electromagnetic tomography (ERP-LORETA) suggests decreased energetic resources for cognitive processing in narcolepsy. Clin Neurophysiol 2008; 119:1782-1794.

Smith JL, Barry RJ, Steiner GZ. CNV resolution does not cause NoGo anteriorisation of the P3: A failure to replicate Simson et al. Int J Psychophysiol 2013; 89:349-357.

Tabachnick BG, Fidell LS. Using Multivariate Statistics, 2nd ed. New York: Harper Collins, 1989.

Tenke CE, Kayser J. Generator localization by current source density (CSD): Implications of volume conduction and field closure at intracranial and scalp locations. Clin Neurophysiol 2012; 123:2328-2345.

Tucker LR. A method for synthesis of factor analysis studies (Personnel Research Section Report No. 984), Washington, D.C.: Department of the Army, 1951.

Volpe U, Mucci A, Bucci P, Merlotti E, Galderisi S, Maj M. The cortical generators of P3a and P3b: A LORETA study. Brain Res Bull 2007; 73:220:230. 


\section{Figure Legends}

Figure 1. Mean ERPs (left column) and corresponding sum of the components identified in the separate PCAs (right column) are shown for Go (black) and NoGo (grey) for the adult (solid trace) and child (dashed trace) groups at the midline sites.

Figure 2. Unscaled PCA factor loadings, factor information, and topographic headmaps (across Go/NoGo conditions) are presented (in colour on the web) for the corresponding components identified in the separate adult (upper panel) and child (lower panel) data. The congruence coefficients $\left(r_{c}\right)$ for each set of corresponding adult and child components are also presented (middle).

Figure 3. Unscaled factor loadings, factor information, and topographic headmaps (across Go/NoGo conditions) from the combined PCA are presented (in colour on the web) for the identified components in the adult (upper panel) and child (lower panel) data. Only components selected for analysis are labelled.

Figure 4. Go and NoGo headmaps (in colour on the web) of the assessed components, drawn from the separate and combined PCAs, are shown for the adults (upper panel), and the children (lower panel).

Figure 5. Major eLORETA sources for the N1-1 component in each condition for adults and children. For each set, major sources are indicated (shown in colour on the web).

Supplementary Figure S1. Major eLORETA sources for all components in each condition for adults and children. For each set, major sources are shown in colour. 
Figure 1.
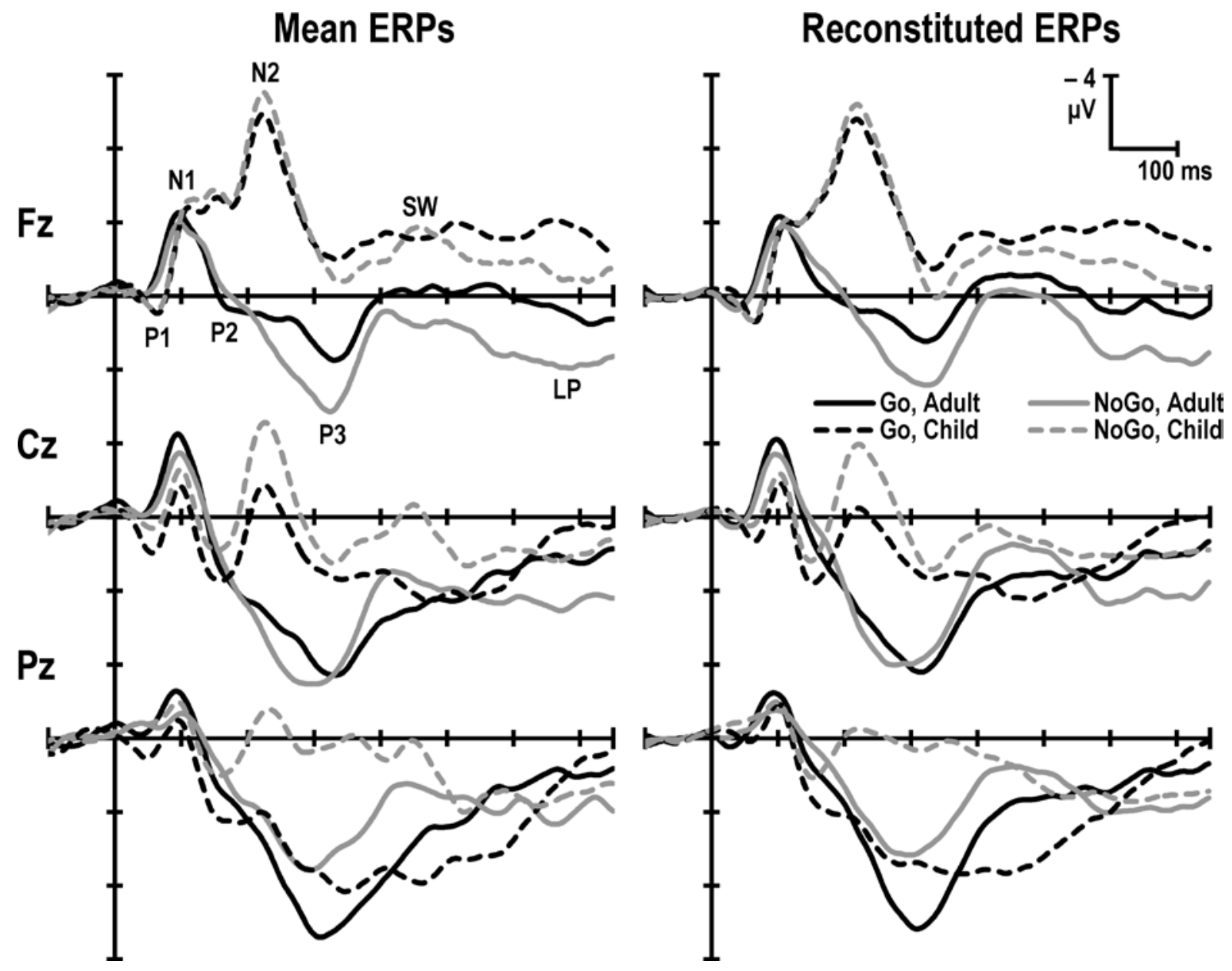
Figure 2.
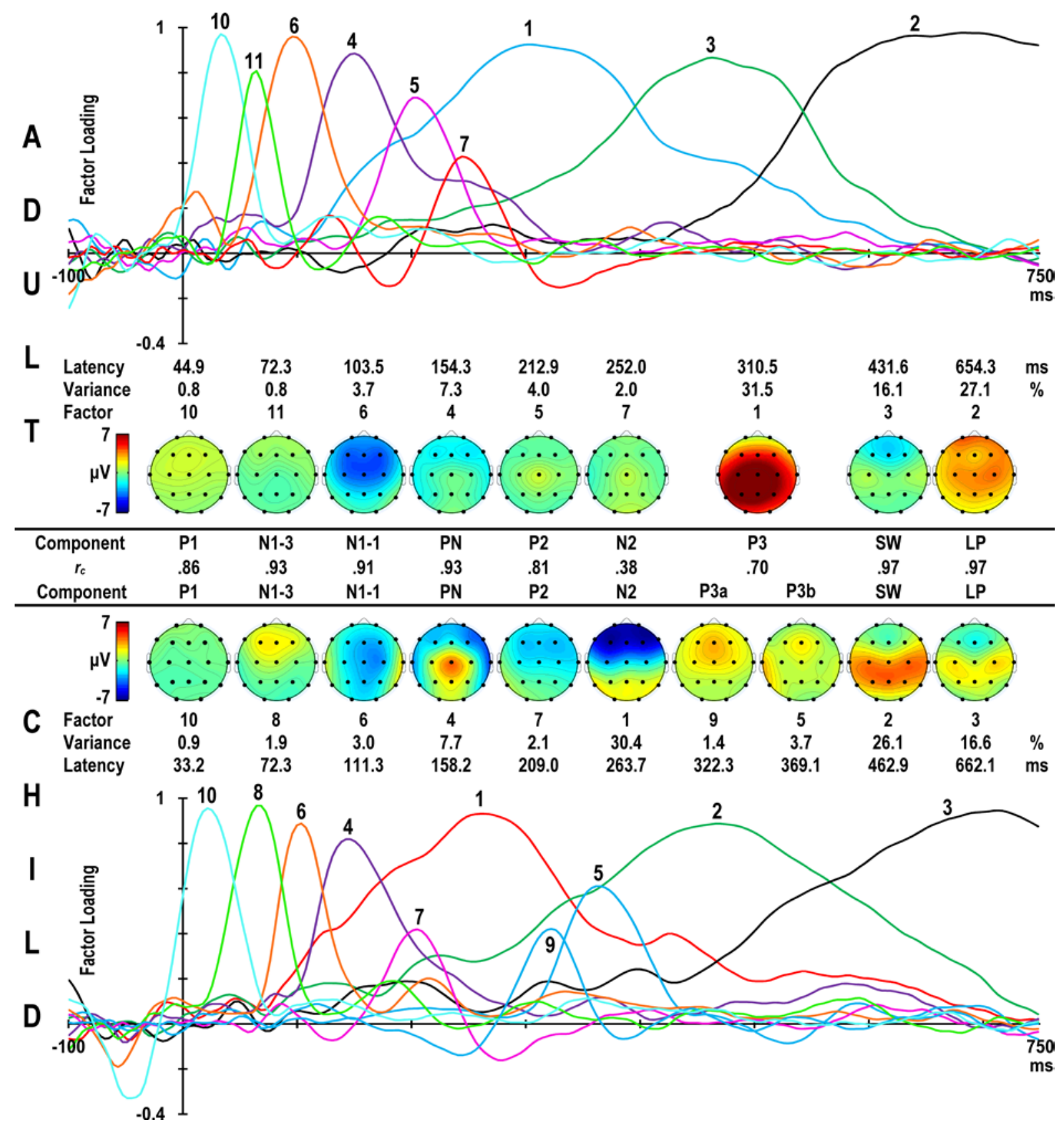
Figure 3.

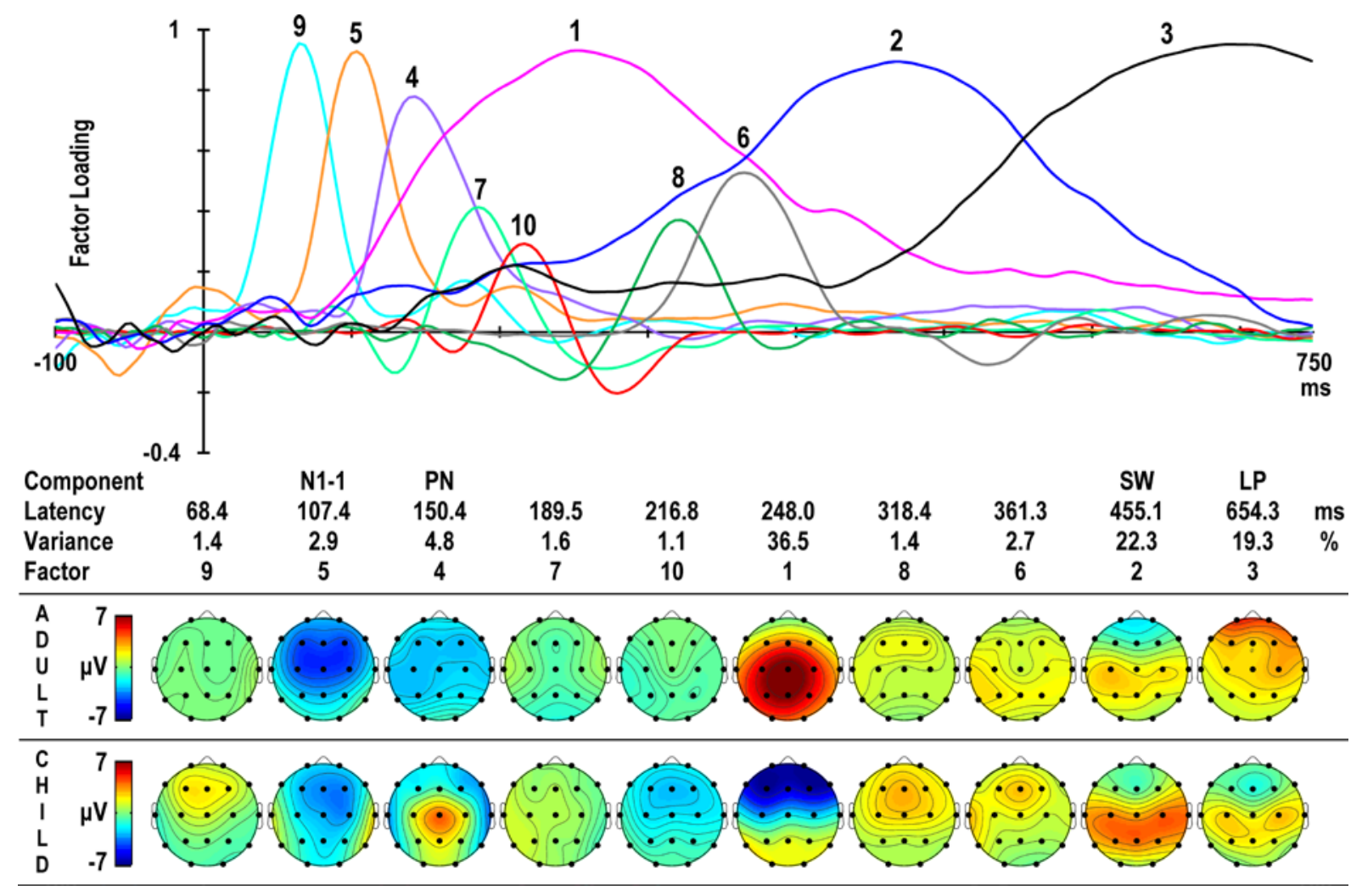

Figure 4.

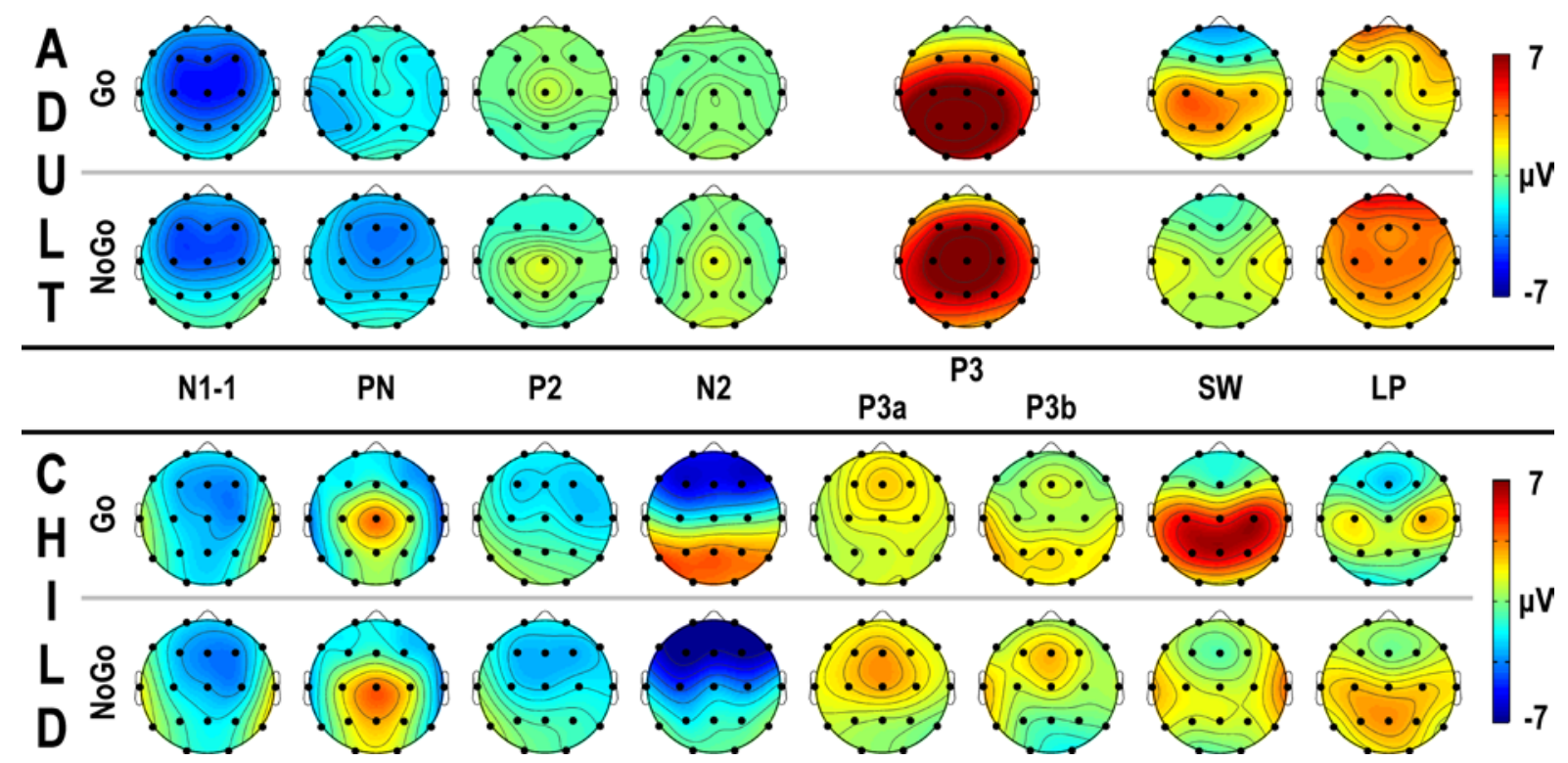


Figure 5.

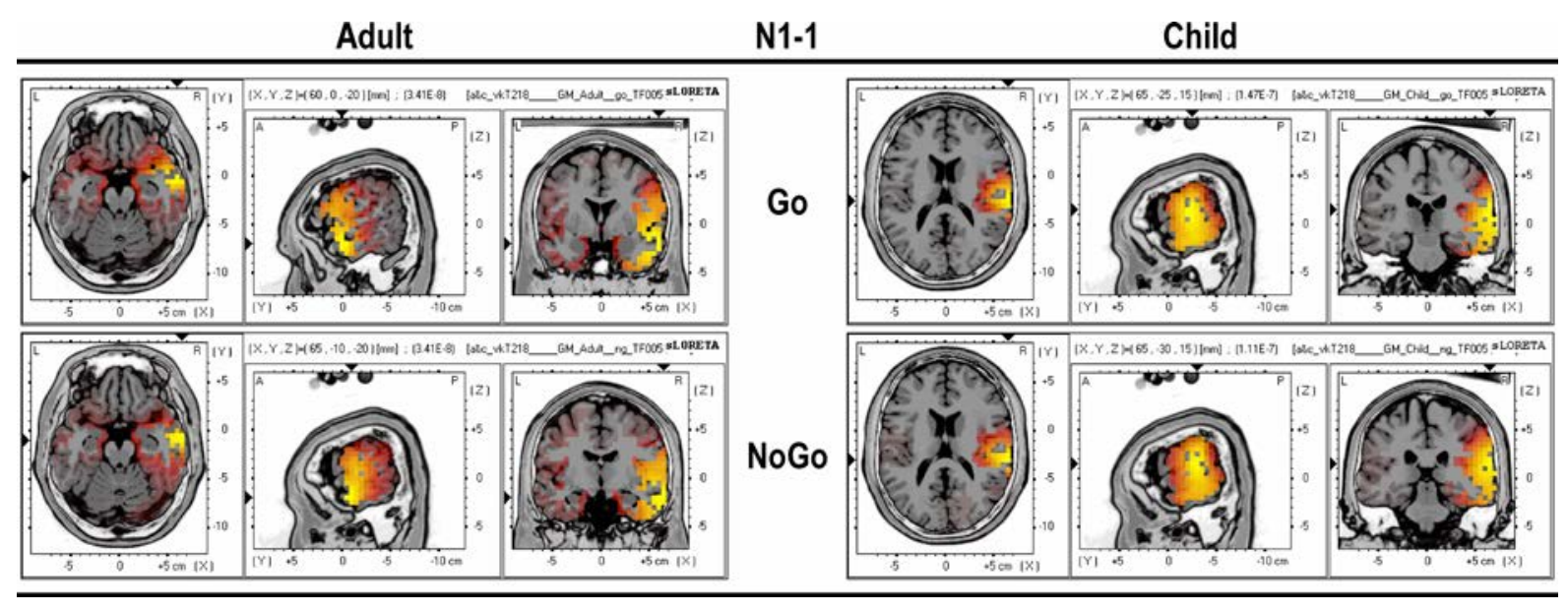

Table 1. Age and performance information $(M[S D])$ for each group, and the statistical relationships between them.

\begin{tabular}{|c|c|c|c|c|c|c|}
\hline & \multirow[b]{2}{*}{ Age (years) } & \multirow[b]{2}{*}{ RT (ms) } & \multicolumn{2}{|c|}{ Errors (\%) } & \multicolumn{2}{|c|}{$\mathrm{N}$ trials } \\
\hline & & & Omission & Commission & Go & NoGo \\
\hline Adult & 20.7 (3.1) & 293.0 (39.9) & $4.1(4.2)$ & $1.5(1.4)$ & $113.8(15.7)$ & $116.6(14.5)$ \\
\hline Child & $10.3(0.5)$ & $368.4(35.5)$ & 14.9 (11.5) & $8.0(7.8)$ & $115.3(45.9)$ & $115.5(47.2)$ \\
\hline$t(\operatorname{adj} . d f)$ & -13.95 (17.9) & $5.98(33.5)$ & $3.74(21.4)$ & $3.48(18.1)$ & $0.14(20.9)$ & $-0.09(20.2)$ \\
\hline$p$ & $<.001$ & $<.001$ & .001 & .003 & .893 & .929 \\
\hline
\end{tabular}

$t$ (adj. $d f)$ : 2-tailed $t$-test ( $d f$ adjusted to reflect unequal variances). 
Table 2. Topographical effects.

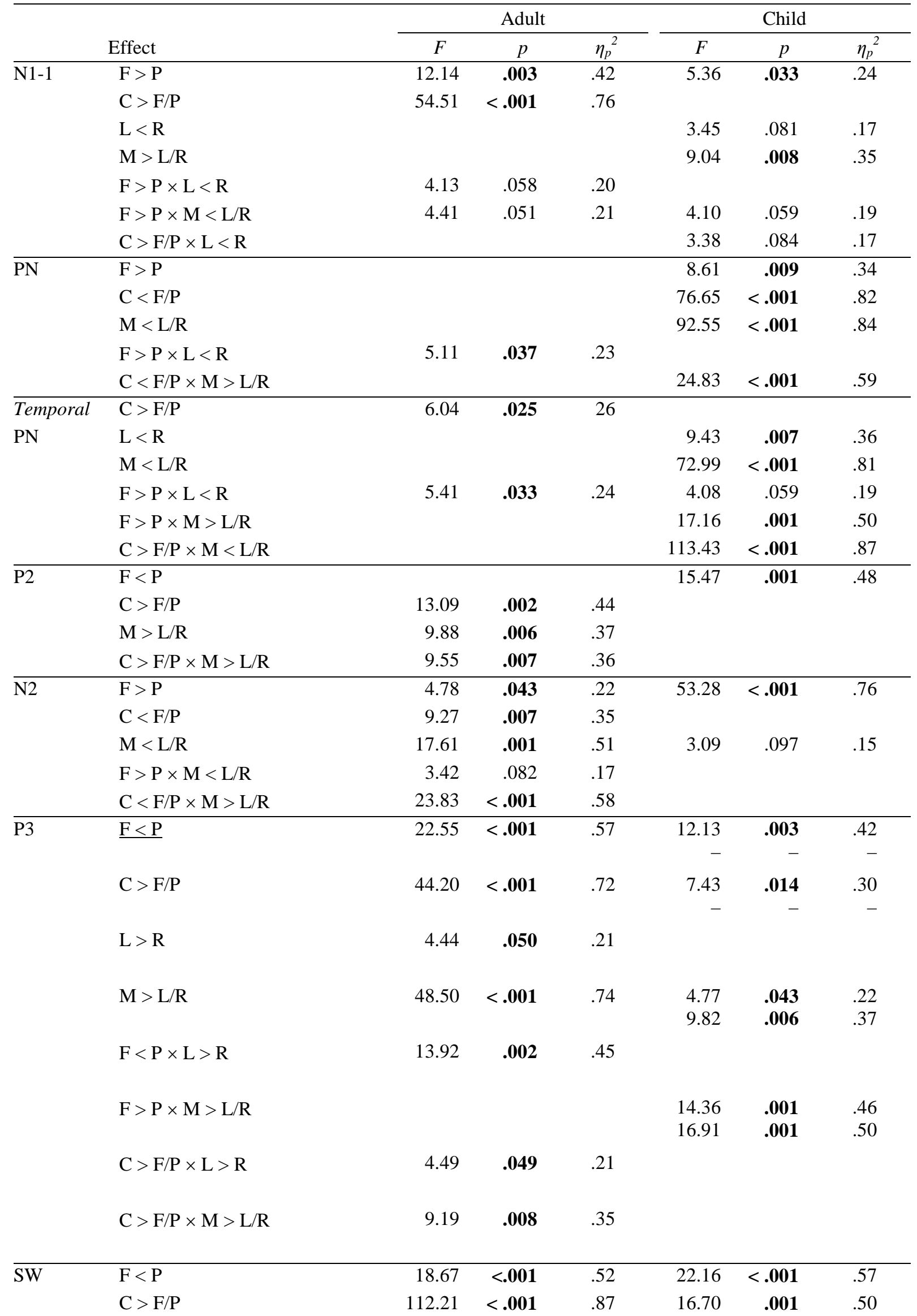




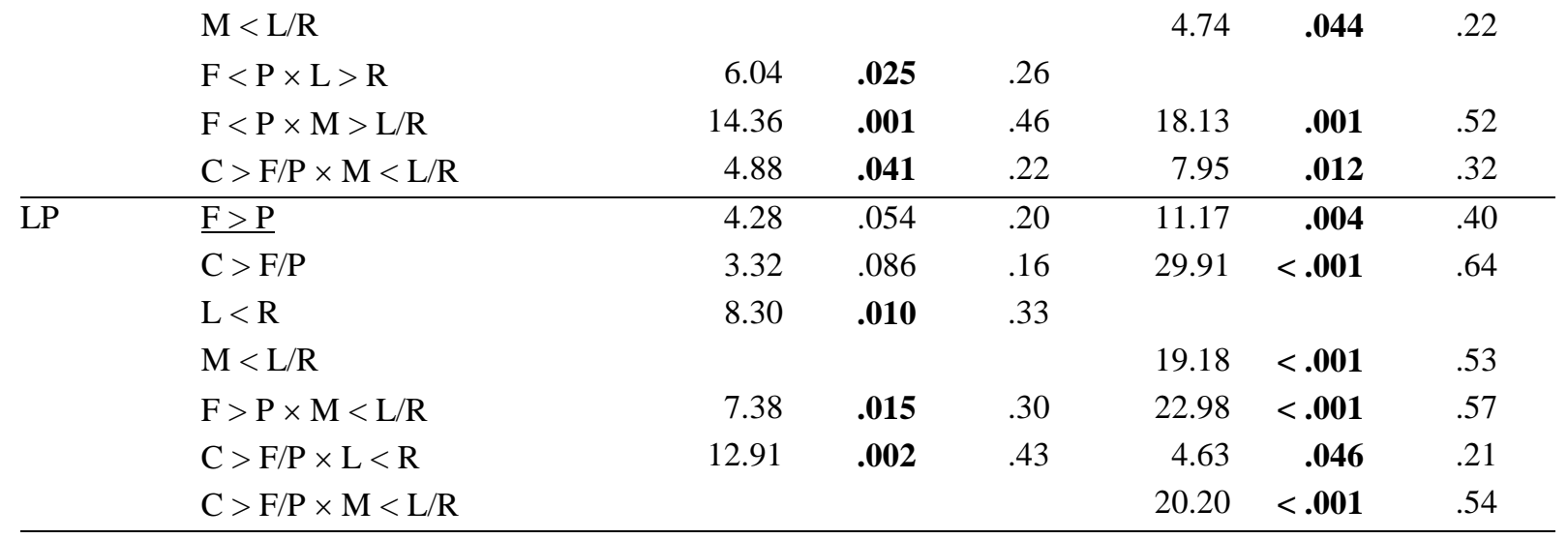

Notes: Child P3 results are presented in component order, with P3a above P3b. Significant effects are indicated by a bold $p$ value. Underlined statistical results indicate a reversal of the corresponding underlined effect or interaction. 
Table 3. Go/NoGo effects.

\begin{tabular}{|c|c|c|c|c|c|c|c|}
\hline \multirow{2}{*}{\multicolumn{2}{|c|}{ Effect }} & \multicolumn{3}{|c|}{ Adult } & \multicolumn{3}{|c|}{ Child } \\
\hline & & $F$ & $p$ & $\eta_{p}^{2}$ & $F$ & $p$ & $\eta_{p}^{2}$ \\
\hline \multirow[t]{3}{*}{$\overline{\mathrm{N} 1-1}$} & Go $>$ NoGo $\times$ F $<$ P & 4.77 & .043 & .22 & & & \\
\hline & Go $>$ NoGo $\times$ M $>$ L/R & 3.89 & .065 & .19 & & & \\
\hline & Go $>$ NoGo & 5.69 & .029 & .25 & & & \\
\hline \multirow[t]{7}{*}{$\overline{\mathrm{PN}}$} & Go $<$ NoGo $\times \mathrm{F}>\mathrm{P}$ & 12.37 & .003 & .42 & 7.26 & .015 & .30 \\
\hline & Go $<$ NoGo $\times$ C $>$ F/P & 3.98 & .062 & .19 & & & \\
\hline & Go $<$ NoGo $\times$ L $<$ R & 5.42 & .032 & .24 & & & \\
\hline & Go $<$ NoGo $\times$ M $>$ L $/ \mathrm{R}$ & 32.94 & $<.001$ & .66 & & & \\
\hline & Go $<$ NoGo $\times$ F $>$ P $\times$ M $>$ L $/ \mathrm{R}$ & 15.65 & .001 & .48 & & & \\
\hline & Go $<$ NoGo $\times$ C $>$ F/P $\times$ M $>$ L/R & 10.21 & .005 & .38 & 5.89 & .027 & .26 \\
\hline & Go $<$ NoGo & 5.68 & .029 & .25 & & & \\
\hline Temporal & Go $<$ NoGo $\times \mathrm{F}>\mathrm{P}$ & 11.79 & .003 & .41 & 3.20 & .092 & .16 \\
\hline \multirow[t]{5}{*}{$\mathrm{PN}$} & Go $<$ NoGo $\times$ L $<$ R & 6.29 & .023 & .27 & & & \\
\hline & Go $<$ NoGo $\times$ M $>$ L/R & 16.21 & .001 & .49 & & & \\
\hline & Go $<$ NoGo $\times$ F $>$ P $\times$ M $>$ L $/ \mathrm{R}$ & 14.81 & .001 & .47 & & & \\
\hline & Go $<$ NoGo $\times$ C $>$ F $/ \mathrm{P} \times \mathrm{M}>\mathrm{L} / \mathrm{R}$ & 19.32 & $<.001$ & .53 & 3.77 & .069 & .18 \\
\hline & Go $<$ NoGo & 3.97 & .063 & .19 & & & \\
\hline \multirow[t]{5}{*}{$\overline{\mathrm{P} 2}$} & Go $<$ NoGo $\times$ F $<$ P & 6.24 & .023 & .27 & & & \\
\hline & Go $<$ NoGo $\times$ C $>$ F/P & 4.92 & .040 & .22 & & & \\
\hline & Go $<$ NoGo $\times$ M $<$ L/R & & & & 7.28 & .015 & .30 \\
\hline & Go $<$ NoGo $\times$ C $>$ F $/ \mathrm{P} \times \underline{\mathrm{L}}>\mathrm{R}$ & 5.44 & .032 & .24 & $\underline{12.55}$ & .003 & .42 \\
\hline & Go $<$ NoGo $\times$ C $>$ F $/ \mathrm{P} \times \mathrm{M}<\mathrm{L} / \mathrm{R}$ & & & & 9.97 & .006 & .37 \\
\hline \multirow[t]{5}{*}{$\mathrm{N} 2$} & Go $>$ NoGo $\times$ F $>$ P & & & & 14.63 & .001 & .46 \\
\hline & Go $>$ NoGo $\times \mathrm{C}>\mathrm{F} / \mathrm{P}$ & 4.24 & .055 & .20 & 3.82 & .067 & .18 \\
\hline & Go $>$ NoGo $\times$ M $>$ L/R & 25.06 & $<.001$ & .60 & & & \\
\hline & Go $>$ NoGo $\times \mathrm{C}>\mathrm{F} / \mathrm{P} \times \mathrm{M}>\mathrm{L} / \mathrm{R}$ & 7.86 & .012 & .32 & 5.32 & .034 & .24 \\
\hline & Go $<$ NoGo & & & & 10.41 & .005 & .38 \\
\hline \multirow[t]{11}{*}{$\overline{\mathrm{P} 3}$} & Go $>$ NoGo $\times$ F $<$ P & 50.27 & $<.001$ & .75 & - & - & - \\
\hline & & & & & 16.27 & .001 & .49 \\
\hline & Go $>$ NoGo $\times \mathrm{C}<\mathrm{F} / \mathrm{P}$ & 4.23 & .055 & .20 & 10.77 & .004 & .39 \\
\hline & & & & & 17.42 & .001 & .51 \\
\hline & $\underline{\mathrm{Go}}>\mathrm{NoGo} \times \mathrm{L}>\mathrm{R}$ & 7.10 & .016 & .29 & $\underline{4.14}$ & .058 & .20 \\
\hline & & & & & - & - & - \\
\hline & Go $>$ NoGo $\times$ F $<$ P $\times$ L $>$ R & 15.12 & .001 & .47 & & & \\
\hline & Go $>$ NoGo $\times \mathrm{C}<\mathrm{F} / \mathrm{P} \times \mathrm{L}>\mathrm{R}$ & 7.76 & .013 & .31 & - & - & - \\
\hline & & & & & 6.14 & .024 & .27 \\
\hline & Go $>$ NoGo $\times$ C $<$ F/P $\times$ M $>$ L/R & 13.06 & .002 & .43 & - & - & - \\
\hline & & & & & 16.42 & .001 & .49 \\
\hline \multirow[t]{7}{*}{$\overline{\mathrm{SW}}$} & Go $>$ NoGo $\times$ F $<$ P & 12.56 & .002 & .42 & 15.31 & .001 & .47 \\
\hline & Go $>$ NoGo $\times$ C $>$ F/P & 36.24 & $<.001$ & .68 & 9.24 & .007 & .35 \\
\hline & Go $>$ NoGo $\times$ L $>$ R & 6.46 & .021 & .28 & & & \\
\hline & Go $>$ NoGo $\times$ M $>$ L/R & 3.33 & .086 & .16 & & & \\
\hline & Go $>$ NoGo $\times \mathrm{F}<\mathrm{P} \times \mathrm{L}>\mathrm{R}$ & 6.61 & .020 & .28 & & & \\
\hline & Go $>$ NoGo $\times \mathrm{F}<\mathrm{P} \times \mathrm{M}>\mathrm{L} / \mathrm{R}$ & 4.10 & .059 & .19 & 5.93 & .026 & .26 \\
\hline & Go $>$ NoGo $\times$ C $>$ F $/ \mathrm{P} \times \mathrm{L}<\mathrm{R}$ & & & & 7.09 & .016 & .29 \\
\hline
\end{tabular}




\begin{tabular}{|c|c|c|c|c|c|c|c|}
\hline & Go $>$ NoGo $\times$ C $>$ F $/ \mathrm{P} \times \mathrm{M}<\mathrm{L} / \mathrm{R}$ & & & & 7.81 & .012 & .31 \\
\hline & Go $>$ NoGo & 4.90 & .041 & .22 & 14.35 & .001 & .46 \\
\hline$\overline{\mathrm{LP}}$ & Go $<$ NoGo $\times$ C $<$ F/P & & & & 9.02 & .008 & .35 \\
\hline & Go $<$ NoGo $\times$ L $>$ R & 25.71 & $<.001$ & .60 & & & \\
\hline & Go $<$ NoGo $\times \mathrm{M}>\mathrm{L} / \mathrm{R}$ & 4.35 & .052 & .20 & 8.66 & .009 & .34 \\
\hline & Go $<$ NoGo $\times$ F $>$ P $\times$ L $>$ R & & & & 3.05 & .099 & .15 \\
\hline & Go $<$ NoGo $\times$ C $>$ F $/ \mathrm{P} \times \mathrm{L}>\mathrm{R}$ & 47.68 & $<.001$ & .74 & 3.93 & .064 & .19 \\
\hline & $\mathrm{Go}<\mathrm{NoGo} \times \mathrm{C}>\mathrm{F} / \mathrm{P} \times \mathrm{M}>\mathrm{L} / \mathrm{R}$ & & & & 7.14 & .016 & .30 \\
\hline & Go $<$ NoGo & 11.38 & .004 & .40 & 5.86 & .027 & .26 \\
\hline
\end{tabular}

Notes: Child P3 results are presented in component order, with P3a above P3b. Significant effects are indicated by a bold $p$ value. Underlined statistical results indicate a reversal of the corresponding underlined effect or interaction. 


\begin{tabular}{|c|c|c|c|c|}
\hline & & 0 & & Go \\
\hline & Adult & Child & Adult & Child \\
\hline N1-1 & $\begin{array}{l}\text { Middle Temporal Gyrus (BA21), Inferior } \\
\text { Temporal Gyrus (BA21), Superior } \\
\text { Temporal Gyrus (BA38) }\end{array}$ & $\begin{array}{l}\text { Superior Temporal Gyrus (BA22, } \\
\text { BA42), Postcentral gyrus (BA40) }\end{array}$ & $\begin{array}{l}\text { Inferior Temporal Gyrus (BA20, BA21), } \\
\text { Middle Temporal Gyrus (BA21) }\end{array}$ & $\begin{array}{l}\text { Superior Temporal Gyrus (BA22, BA42), } \\
\text { Postcentral Gyrus (BA40) }\end{array}$ \\
\hline PN & $\begin{array}{l}\text { Fusiform Gyrus (BA20), Inferior Temporal } \\
\text { Gyrus (BA20, BA37) }\end{array}$ & $\begin{array}{l}\text { Precentral Gyrus (BA4, BA6), Middle } \\
\text { Frontal Gyrus (BA6) }\end{array}$ & Precuneus (BA7, BA19), Cuneus (BA19) & $\begin{array}{l}\text { Postcentral Gyrus (BA1, BA3), Precentral } \\
\text { Gyrus (BA4) }\end{array}$ \\
\hline P2 & $\begin{array}{l}\text { Postcentral Gyrus (BA5), Paracentral } \\
\text { Lobule (BA3, BA4) }\end{array}$ & $\begin{array}{l}\text { Superior Temporal Gyrus (BA38), Orbital } \\
\text { Gyrus (BA11), Inferior Frontal Gyrus } \\
\text { (BA11) }\end{array}$ & Postcentral Gyrus (BA1, BA2, BA5) & $\begin{array}{l}\text { Medial Frontal Gyrus (BA11), Subcallosal } \\
\text { Gyrus (BA25), Fusiform Gyrus (BA20) }\end{array}$ \\
\hline N2 & $\begin{array}{l}\text { Medial Frontal Gyrus (BA11), Superior } \\
\text { Frontal Gyrus (BA11), Rectal Gyrus } \\
\text { (BA11) }\end{array}$ & $\begin{array}{l}\text { Postcentral Gyrus (BA3), Middle Frontal } \\
\text { Gyrus (BA8), Sub-Gyral (BA2) }\end{array}$ & $\begin{array}{l}\text { Postcentral Gyrus (BA3), Precentral } \\
\text { Gyrus (BA4, BA6) }\end{array}$ & $\begin{array}{l}\text { Postcentral Gyrus (BA40), Middle } \\
\text { Temporal Gyrus (BA39), Superior } \\
\text { Temporal Gyrus (BA22) }\end{array}$ \\
\hline P3 & $\begin{array}{l}\text { Rectal Gyrus (BA11), Inferior Frontal } \\
\text { Gyrus (BA47), Medial Frontal Gyrus } \\
\text { (BA25) }\end{array}$ & & $\begin{array}{l}\text { Medial Frontal Gyrus (BA11, BA25), } \\
\text { Anterior Cingulate (BA32) }\end{array}$ & \\
\hline P3a & & $\begin{array}{l}\text { Superior Frontal Gyrus (BA6), Cingulate } \\
\text { Gyrus (BA32), Medial Frontal Gyrus } \\
\text { (BA9) }\end{array}$ & & $\begin{array}{l}\text { Medial Frontal Gyrus (BA6), Superior } \\
\text { Frontal Gyrus (BA6), Paracentral Lobule } \\
\text { (BA31) }\end{array}$ \\
\hline P3b & & $\begin{array}{l}\text { Inferior Frontal Gyrus (BA47), Superior } \\
\text { Temporal Gyrus (BA38), Parahippocampal } \\
\text { Gyrus (BA19) }\end{array}$ & & $\begin{array}{l}\text { Fusiform Gyrus (BA37), Middle Frontal } \\
\text { Gyrus (BA11), Superior Frontal Gyrus } \\
\text { (BA11) }\end{array}$ \\
\hline SW & $\begin{array}{l}\text { Anterior Cingulate (BA32), Medial } \\
\text { Frontal Gyrus (BA9, BA32) }\end{array}$ & $\begin{array}{l}\text { Superior Frontal Gyrus (BA6), Medial } \\
\text { Frontal Gyrus (BA6), Middle Frontal } \\
\text { Gyrus (BA6) }\end{array}$ & $\begin{array}{l}\text { Inferior Frontal Gyrus (BA45, BA47), } \\
\text { Precentral Gyrus (BA44) }\end{array}$ & $\begin{array}{l}\text { Inferior Temporal Gyrus (BA20), Fusiform } \\
\text { Gyrus (BA37), Middle Temporal Gyrus } \\
\text { (BA37) }\end{array}$ \\
\hline LP & $\begin{array}{l}\text { Medial Frontal Gyrus (BA10), Superior } \\
\text { Frontal Gyrus (BA10), Middle Frontal } \\
\text { Gyrus (BA10) }\end{array}$ & $\begin{array}{l}\text { Middle Frontal Gyrus (BA8, BA9), } \\
\text { Precentral Gyrus (BA9) }\end{array}$ & $\begin{array}{l}\text { Medial Frontal Gyrus (BA10, BA11), } \\
\text { Superior Frontal Gyrus (BA10) }\end{array}$ & $\begin{array}{l}\text { Superior Temporal Gyrus (BA22, BA42), } \\
\text { Middle Temporal Gyrus (BA22) }\end{array}$ \\
\hline
\end{tabular}

Corresponding adult/child structures are indicated in bold. 

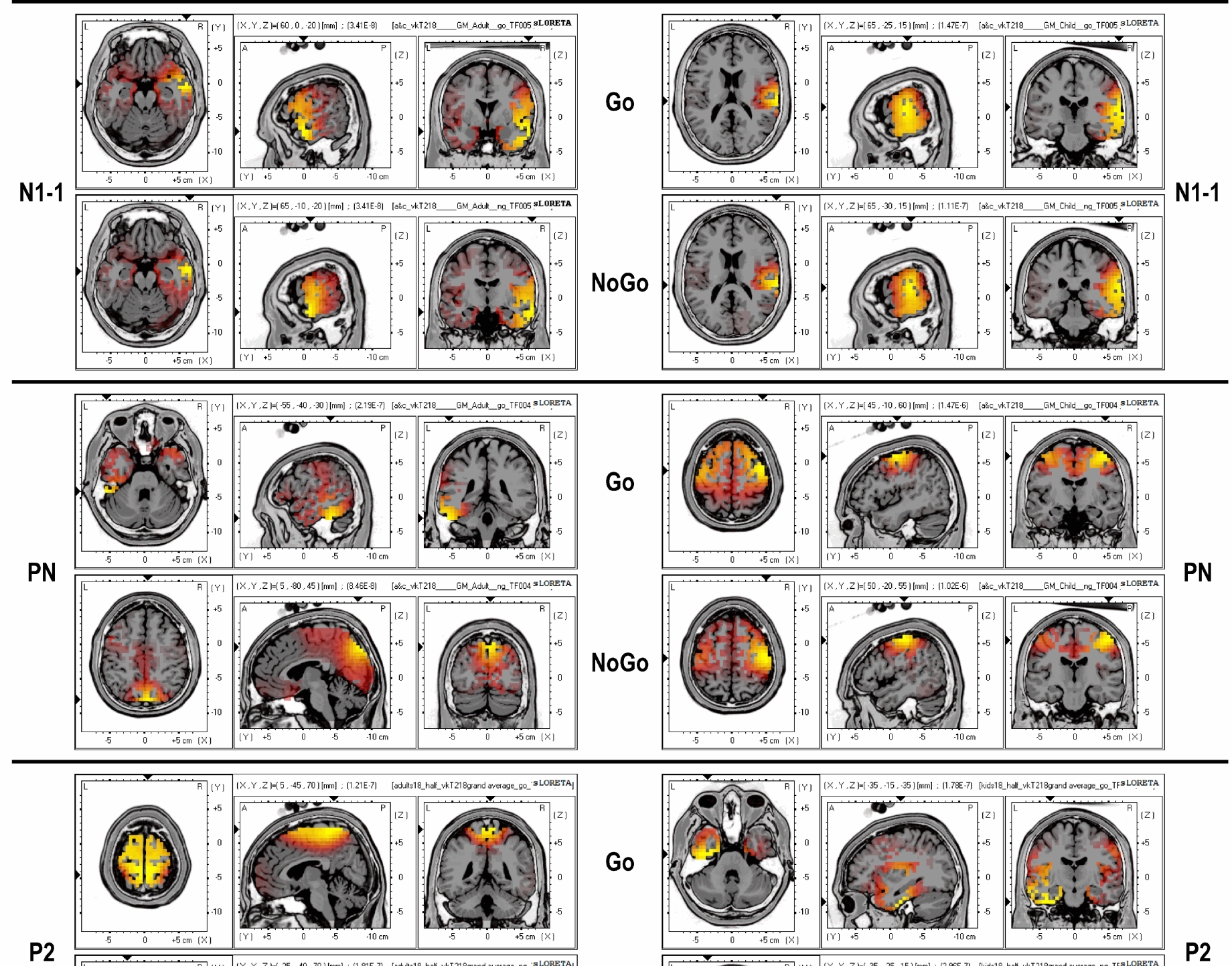

P2
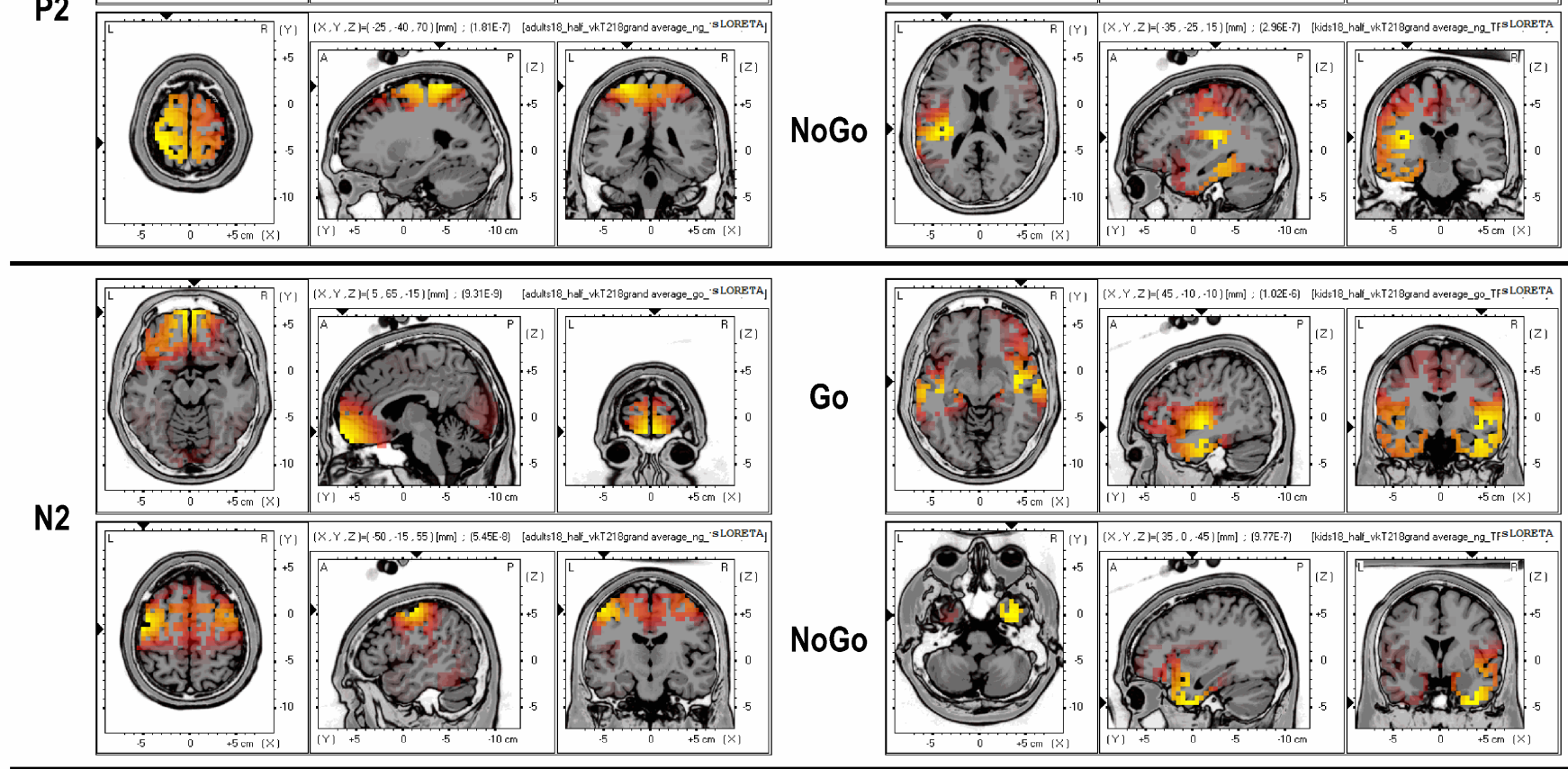


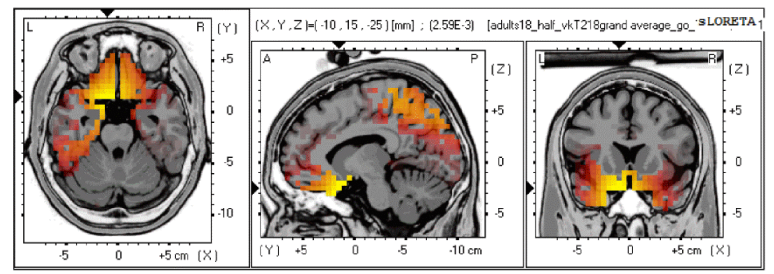

\section{Go}

P3
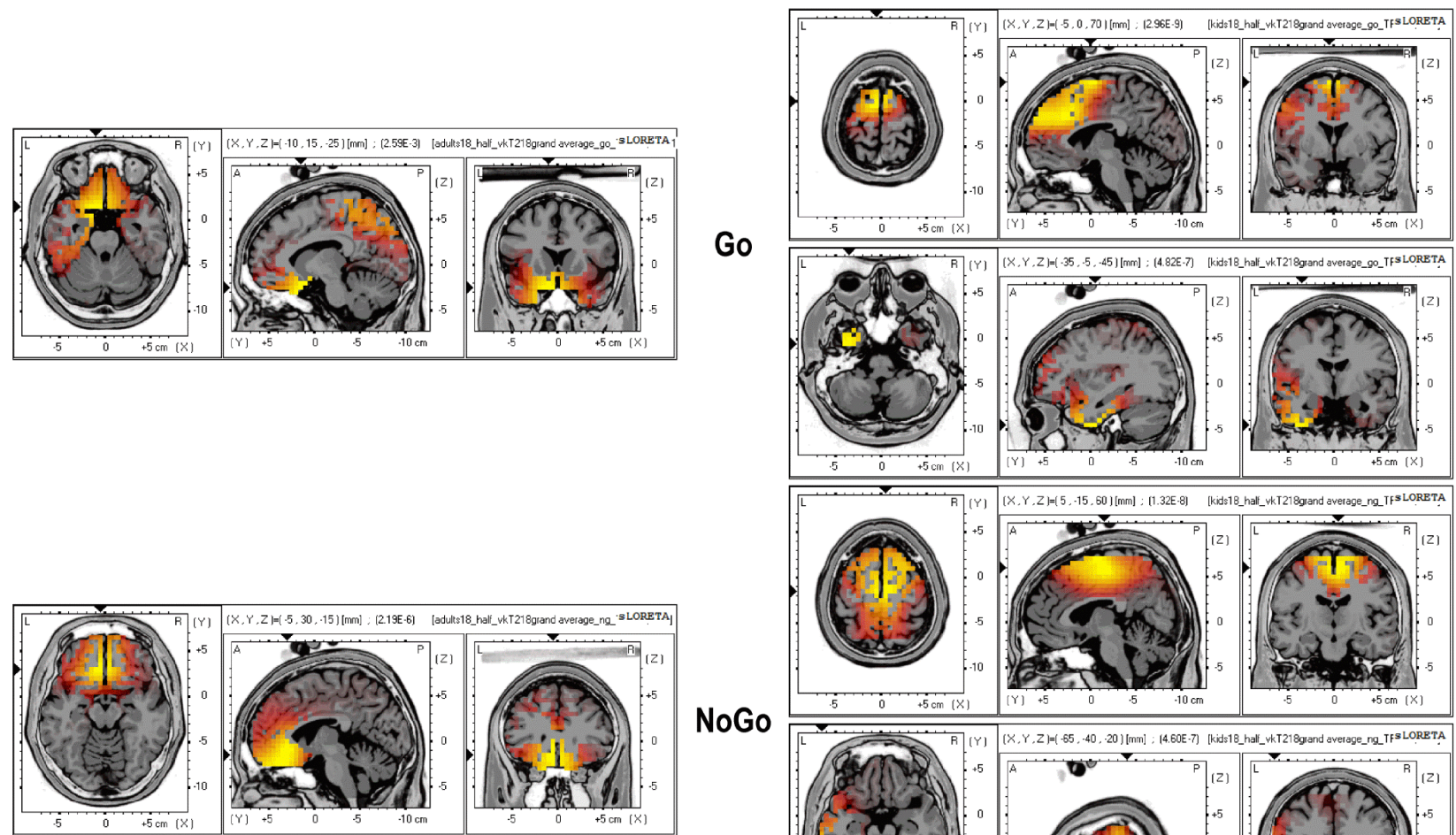

NoGo
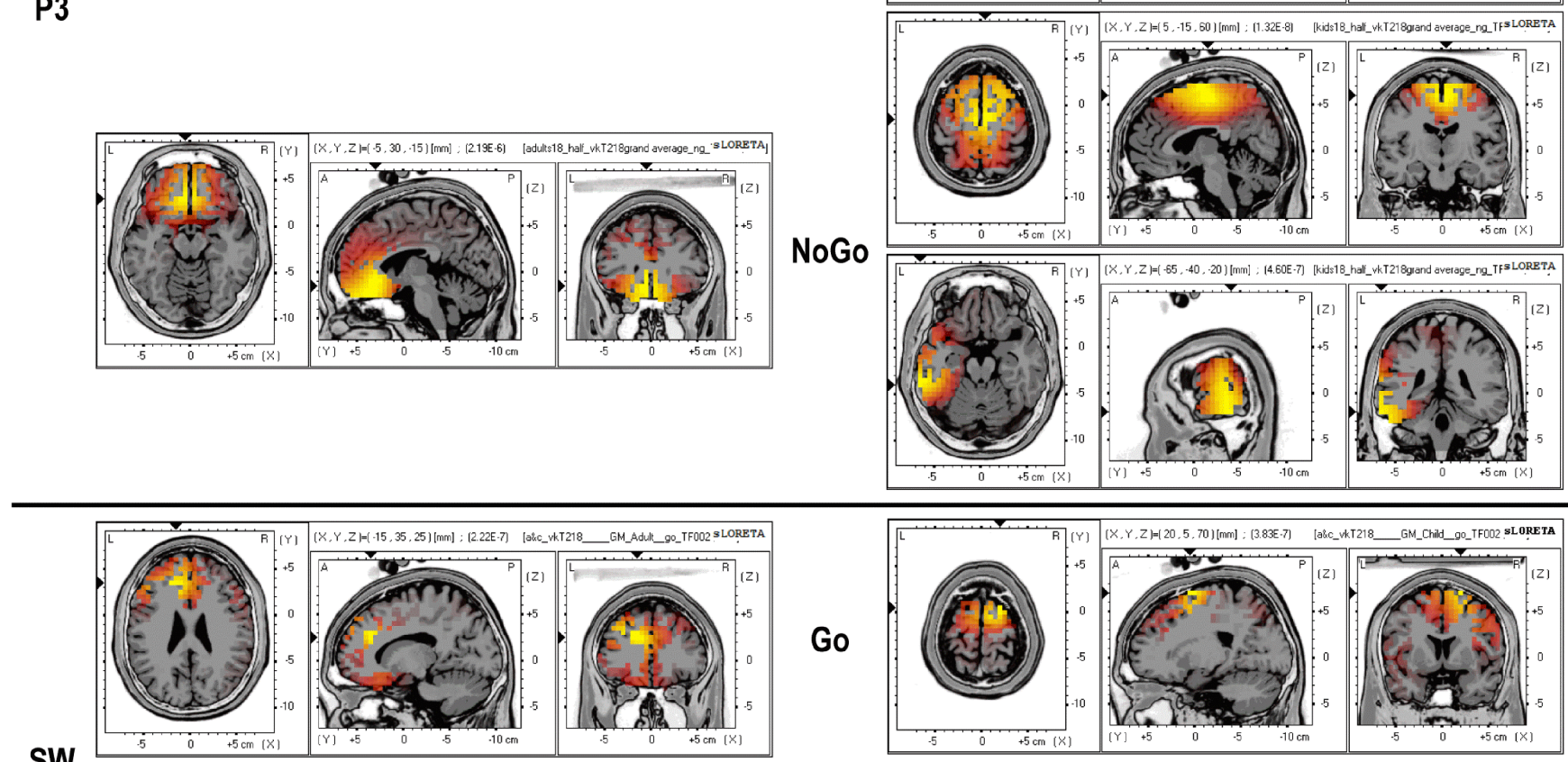

SW
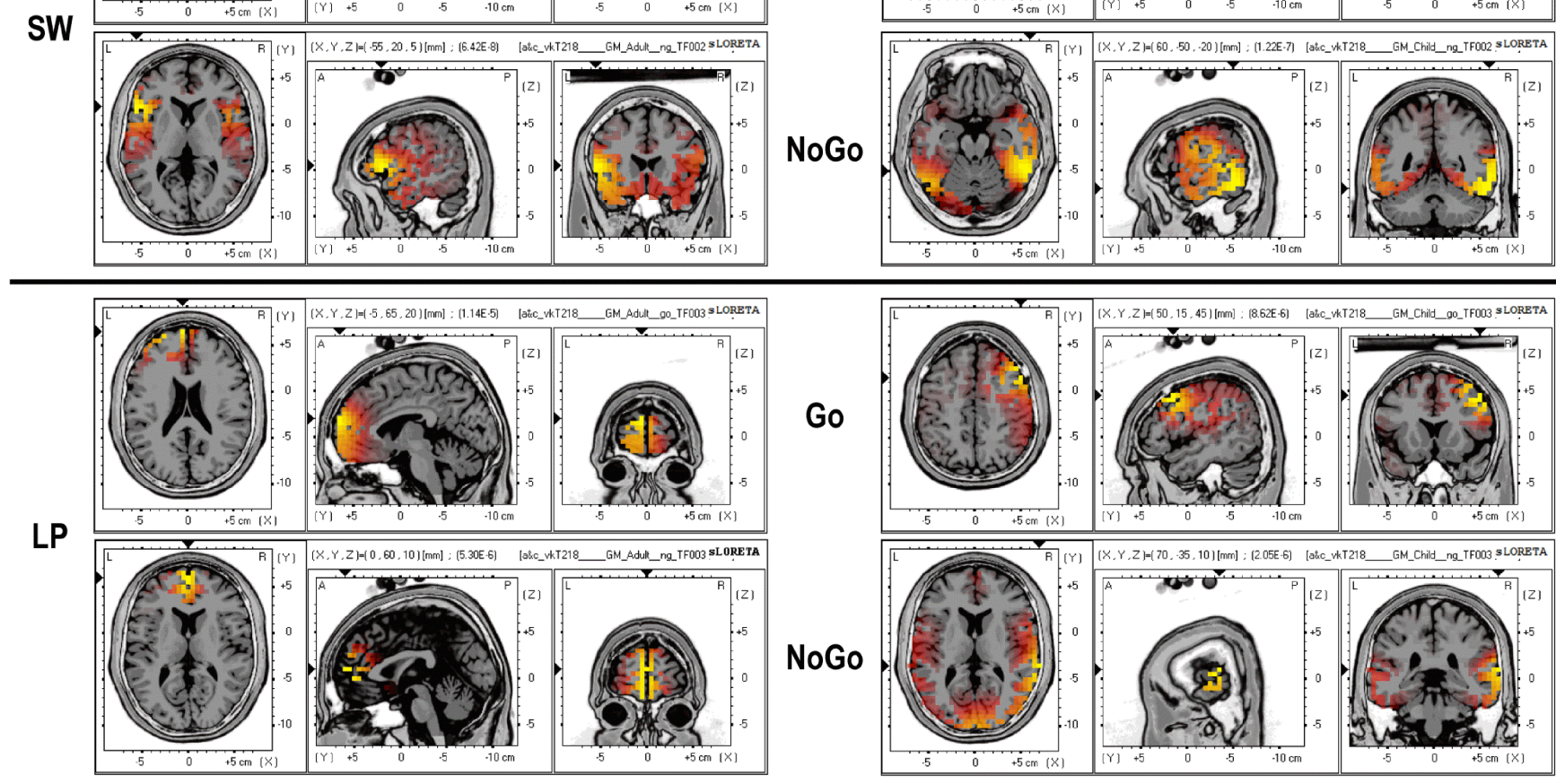

Supplementary Figure S1. Major eLORETA sources for all components in each condition for adults and children. For each set, major sources are shown in colour. 